\title{
Predicting the airborne microbial transmission via human breath particles using a Gated Recurrent Units neural network
}

\begin{tabular}{|r|l|}
\hline Journal: & International Journal of Numerical Methods for Heat and Fluid Flow \\
\hline Manuscript ID & HFF-07-2021-0498.R1 \\
\hline Manuscript Type: & Research Article \\
\hline Keywords: & $\begin{array}{l}\text { COVID-19 infection, CFD modelling, Particle tracking, Inhalation airflow, } \\
\text { Gated Recurrent Units Neural Network }\end{array}$ \\
\hline \multicolumn{2}{|l}{} \\
\hline
\end{tabular}

\section{SCHOLARONE \\ Manuscripts}




\section{Predicting the airborne microbial transmission via human breath particles using a Gated Recurrent Units neural network}

Abstract

Purpose

The main purpose of this paper is to devise a tool, based on Computational Fluid Dynamics (CFD) and Machine Learning (ML), for the assessment of potential airborne microbial transmission in enclosed spaces. A Gated Recurrent Units Neural Network (GRU-NN) is presented to learn and predict the behaviour of droplets expelled through breaths via particle tracking datasets.

\section{Design/methodology/approach}

A computational methodology is used for investigating how infectious particles originated in one location are transported by air and spread throughout a room. High-fidelity prediction of indoor air flow is obtained by means of an in-house parallel CFD solver which employs a one equation Spalrat-Allmaras (SA) turbulence model. Several flow scenarios are considered by varying different ventilation conditions and source locations. The CFD model is used for computing the trajectories of the particles emitted human breath. The numerical results are used to the ML training.

\section{Finding}

In this work, it is shown that the developed ML model, based on the Gated Recurrent Units Neural Network (GRU-NN), can accurately predict the airborne particle movement across an indoor environment for different vent operation conditions and source locations. The numerical results in the paper prove that the presented methodology is able to provide accurate predictions of the time evolution of particle distribution at different locations of the enclosed space.

\section{Originality/value}

This study paves the way for the development of efficient and reliable tools for predicting virus airborne movement under different ventilation conditions and different human positions within an indoor environments, potentially leading to new design. A parametric study is carried out to evaluate 
the impact of system settings on the time variation particles emitted human breath within the space considered.

Keywords: COVID-19 infection, CFD modelling, Spalrat-Allmaras (SA) model, Particle tracking, Inhalation airflow, Recurrent Neural Network, Gated Recurrent Units (GRU)

\section{Introduction}

In indoor environments, the main transmission route of COVID-19 involves the emission of respiratory droplets from the mouth and nose which can remain suspended in the air for several minutes, exposing the surrounding people to high infection risk [1-4]. In this context, different methodologies for characterizing the fluid dynamics patterns within the indoor environment have been proposed [5] 8 . These efforts have also been accompanied by recent research focusing on how pollution and biological agents can spread throughout an enclosed space [9 14. Recently, Vuorinen, et al. [15] modelled physical processes related to aerosol dispersion in air and focused on transmission by inhalation in the context of COVID-19. These authors gave various examples on the transport and dilution of aerosol dimeters of $d \leq 20$ $\mu m$ over distances $O(10 \mathrm{~m})$ in public indoor environments by Monte-Carlo modelling. Furthermore, Löhner et al. [16, 17] studied the characteristics of virus contaminants and the transmission via droplets and aerosols in a narrow corridor with moving pedestrians and in a typical hospital rooms considering a bi-directional coupling, whereby the flow and the motion of the crowd are computed concurrently and with mutual influences. In subsequent work, Abuhegazy et al.[18] investigated aerosol removal and surface deposition in a realistic classroom with nine students and a teacher using computational fluid particle dynamics algorithm implemented by Ansys Fluent. These authors [18] found that a $24 \%-50 \%$ of particles smaller than $15 \mu \mathrm{m}$ exit the system within 15 minutes through the air conditioning system and particles larger than $20 \mu \mathrm{m}$ almost entirely deposit on the ground, desks, and nearby surfaces in the room. Additionally Lau et al. [19, 20] described a model for indoors airborne transmission where the concentration of airborne infectious particles governed by an advection-diffusion-reaction equation. These authors compared the model both with more complex models and with experimental data and found good agreement. Moreover, to address the relevant background, the impact of ventilation on the airflow pattern has been also extensively studied [21 23]. Ventilation plays an important role in reducing the risk of transmission through dilution and removal of the infected particles within the indoor environment [24].

Despite aerosol transport within indoor environments has been extensively studied in the last decades [25], there is a pressing need for the establishment of efficient computational tools for the prediction of transmission 
and infectivity level of airborne viruses (such as COVID-19 virus) throughout enclosed spaces. To the authors' knowledge, ML methods and specifically Gated Recurrent Units (GRU) neural network [26, 27] have not been employed before for this purpose. With all the information available regarding the migration of airborne infectious particles in indoor environments, a robust control tool to capture, store and analyse data using ML algorithms is essential. Machine Learning represents an efficient and accurate approach to find patterns in the most complex and abstract data by proposing alternatives to analysing large volume of data to forward-looking predictive models [28, 29. ML has attracted strong interest over many years and is a standard tool today in many applied science topics. The main advantage of ML lies in that the computer can achieve the purpose of self-learning and predict the trend through operating algorithms. Because of this feature, the computer can be continuously trained, the training dataset can be increased, and over time more accurate results can be obtained through data accumulation by developing fast and efficient algorithms 30 33].

Through the present study we devised a combined ML and CFD modelling approach for defining the particle distribution associated to airflow patterns within an indoor environment. This includes natural circulation inside enclosed spaces by air-conditioning with several flow scenarios regarding the operation of inlet vents, location of a person in an active office 34 with different human standing positions, analysing the potential of virus spread through the air from an infected person, identification of critical points, and particle dispersion and deposition in the enclosed environment. Moreover, a Gated Recurrent Units Neural Network (GRU-NN) is presented to learn and predict the behaviour of droplets expelled through breaths via particle tracking datasets. This article is organised as follows: Section 2 provides the details on the adopted computational methodology including the particle tracking scheme for modelling infectious particle behaviours. The description of the problem considered is reported in Section 3. The neural network architecture methodology is described in Section 4. This is followed by the Section 5 in which the results are reported. In the last section, the significant findings of the study are summarised.

\section{Mathematical modelling and solution method}

The forced air circulation within an active office may be described by using turbulent incompressible flow equations. These equations and their solution method are briefly summarised in this section.

\subsection{Air flow within the room}

The motion of the fluid throughout the room is described by means of the incompressible Navier-Stokes equations, combined with a turbulence 
model. The mass and momentum conservation equations in dimensional form read:

$$
\begin{aligned}
& \nabla \cdot \boldsymbol{v}=0 \\
& \frac{\partial \boldsymbol{v}}{\partial t}=-(\boldsymbol{v} \cdot \nabla) \boldsymbol{v}-\frac{1}{\rho} \nabla p+\left(\nu+\nu_{T}\right) \nabla^{2} \boldsymbol{v},
\end{aligned}
$$

where $\boldsymbol{v}$ is the velocity vector, $\rho$ is the air density, $p$ is the pressure, $\nu$ is the kinematic viscosity, whilst $\nu_{T}$ is the turbulent eddy viscosity. The space and time distribution of the turbulent eddy viscosity $\nu_{T}$ is obtained by employing the one equation Spalart-Allmaras (SA) model [35]38], which uses several turbulence parameters $\left(c_{b 1}, \sigma, c_{b 2}, k, c_{w 1}, c_{w 2}, c_{w 3}\right.$ and $\left.c_{v 1}\right)$ for describing the transport of the variable $\hat{\nu}=\nu_{T} / f_{v 1}$. The scalar equation is:

$$
\frac{\partial \hat{\nu}}{\partial t}=-\boldsymbol{v} \cdot \nabla \hat{\nu}+c_{b 1} \hat{S} \hat{\nu}+\frac{1}{\sigma}\left[\nabla \cdot((\nu+\hat{\nu}) \nabla \hat{\nu})+c_{b 2}(\nabla \hat{\nu})^{2}\right]-c_{w 1} f_{w}\left[\frac{\hat{\nu}}{y}\right]^{2}
$$

where

$$
\begin{aligned}
& \hat{S}=S+f_{v 2} \frac{\hat{\nu}}{k^{2} y^{2}} \\
& f_{v 2}=1-X /\left(1+X f_{v 1}\right), \\
& f_{v 1}=X^{3} /\left(X^{3}+c_{v 1}^{3}\right), \\
& f_{w}=g\left[\frac{1+c_{w 3}^{6}}{g^{6}+c_{w 3}^{3}}\right]^{1 / 6}, \\
& X=\hat{\nu} / \nu \\
& g=r+c_{w 2}\left(r^{6}-r\right), \\
& r=\frac{\hat{\nu}}{\hat{S} k^{2} y^{2}}
\end{aligned}
$$

in which $S$ is the magnitude of vorticity and $y$ is the near wall distance. The turbulence parameters are set as follows: $c_{b 1}=0.1355, \sigma=2 / 3, c_{b 2}=$ $0.622, k=0.41, c_{w 1}=c_{b 1} / k^{2}+\left(1+c_{b 2}\right) / \sigma, c_{w 2}=0.3, c_{w 3}=2$ and $c_{v 1}=7.1$.

The equations above are solved by using an in-house parallel CFD library based on a established finite-element characteristic-based split (CBS) scheme, which is suitable for problems with unstructured meshes [37, 39]. The velocity and pressure fields within the room are computed in time by solving Equations 1, 2, in conjunction with the SA turbulence model (Equation 3). Here we used the semi-implicit (in time) version of the CBS since it represent a good compromise between efficiency, accuracy and flexibility for external component integration [40]. These features make the scheme ideal for computing incompressible flow in complex geometries. The code is written in Fortran90 and all simulations are carried out using an Open shift Container Platform (OCP) cluster on 40 processors with the OpenMPI. 


\subsection{Particle tracking}

To calculate the trajectory of a particle drifted by the air current in the room, we evaluate the particle pathway in every mesh cell met by the particle during its travel. Since we use a tetrahedral mesh, the calculation of trajectory requires tracing a particle through a tetrahedron. Here, it is necessary to calculate the leaving point in the tetrahedron surface for given entering point alongside the propagation time trough the cell [25]. For this, we use the linear shape functions $\xi_{i}$ associated to the nodes of the tetrahedron. In this way, any point $\boldsymbol{P}$ inside the cell can be determined as a linear combination of the coordinates of the tetrahedron vertices $\boldsymbol{P}_{i}$ :

$$
\boldsymbol{P}=\sum_{i=1}^{4} \xi_{i} \boldsymbol{P}_{i}
$$

where $\sum_{i=1}^{4} \xi_{i}(\boldsymbol{P})=1$.

Here the velocities are computed at cell vertices, and the velocity varies linearly in space over every cell. The velocity at any point $\boldsymbol{P}$ can be expressed through its shape functions

$$
\boldsymbol{v}=\sum_{i=1}^{4} \xi_{i} \boldsymbol{v}_{i}
$$

where $\boldsymbol{v}_{i}$ are velocities at tetrahedron vertices. By solving a system of three linear equations we can represent velocity $\boldsymbol{v}$ at $\boldsymbol{P}$ through coordinates of tetrahedron vertices in the following form

$$
\boldsymbol{v}=\sum_{i=1}^{4} \hat{v}_{i} \boldsymbol{P}_{i}, \quad \sum_{i=1}^{4} \hat{v}_{i}=0
$$

The linear shape functions $\xi_{i}$ also can be treated as coordinates in the master element. Then every parameter $\hat{v}_{i}$ in 13 represents a velocity component along $i$ th master element coordinate $\xi_{i}$. This enables calculation of the propagation time of the particle to the plane of every face and finding which face can be reached first, i.e. the face containing the leaving point.

A trajectory in the linearly varying velocity field can be expressed analytically through the exponential function, but to find the leaving point we have to solve numerically an algebraic equation (see [25]). Instead we propose here a fast and accurate predictor-corrector type method. First, we calculate the values of shape functions for the entering point $\boldsymbol{P}^{\text {in }}$ and, employing equation 12 , calculate the velocity vector $\boldsymbol{v}^{\text {in }}$ at it. Considering velocity $\boldsymbol{v}^{\text {in }}$ as the uniform velocity, we calculate the master element velocity components 13 and the predicted leaving point $\boldsymbol{P}_{*}^{\text {out }}$. Second, we calculate the velocity $\boldsymbol{v}_{*}^{\text {out }}$ at this predicted leaving point. Now we take the mean velocity

$$
\boldsymbol{v}=\frac{1}{2}\left(\boldsymbol{v}^{\text {in }}+\boldsymbol{v}_{*}^{\text {out }}\right)
$$


and considering it as a uniform velocity in the cell, calculate the master element velocity components and the corrected leaving point $\boldsymbol{P}^{\text {out }}$ as well as the propagation time.

The leaving point of the given cell is an entering point of the adjacent cell in which this algorithm is repeated. As the result, the trajectory of particle is determined by entering/leaving points at the boundary between two adjacent cells and time instants when these points are reached. After that the trajectory is linearly interpolated onto a uniform time grid.

The trajectory can be terminated

1. if the particle leaves the domain through the outlet (no adjacent cell at the leaving cell face);

2. if the particle is settled at a wall or other surface: floor, ceiling, pipeline, etc. This can occur if the particle reaches a near-boundary cell which has three boundary vertices and the velocity at the nonboundary vertex has a component toward the boundary;

3 . if the particle is trapped in the air between two adjacent cells having mean velocities directed to each other. This can happen near stagnation points in the velocity field: intersection of three flow separation surfaces. In a real flow, the probability to get into such point is infinitesimal. In a discretised domain used for computation, this probability is small but finite.

4. Some particles can remain in the air for a long time trapped by a large vortex caused by intensive ventilation.

The algorithm has been implemented in $\mathrm{C}++$. Computation of several hundred trajectories with the maximal preset time of one hour is performed in less than one second.

\section{Problem specification}

We considered an indoor space within Swansea University Bay Campus, part of a building constructed in 2018 for studying the performances of an active office [34]. The air conditioning system in active office consists of two supply diffusers and one door vent as shown in Figure 1. In this work, and for the computational modelling, a human body is placed under vent2 (case 1) and at the middle between two vents (case 2) of a $46.44 \mathrm{~m}^{3}$ room $4.0 \mathrm{~m}$ by $4.3 \mathrm{~m}$, and $2.7 \mathrm{~m}$ high. Additionally, six different human standing positions $(0,60,120,180,240$, and 300 degrees $)$ are considered. Figure 1 illustrates the configuration of the simulated room. Detailed characteristics for each mesh is recorded in Table 1 for case 1 (human body under vent2). Almost the same mesh characteristics are used for case 2 (not shown). Extensive mesh sensitivity for the characteristic-based split (CBS) method has been performed in previous articles (see for example, [41, 42]). The Reynolds number for this study is defined based on the vents diameter of the room. 

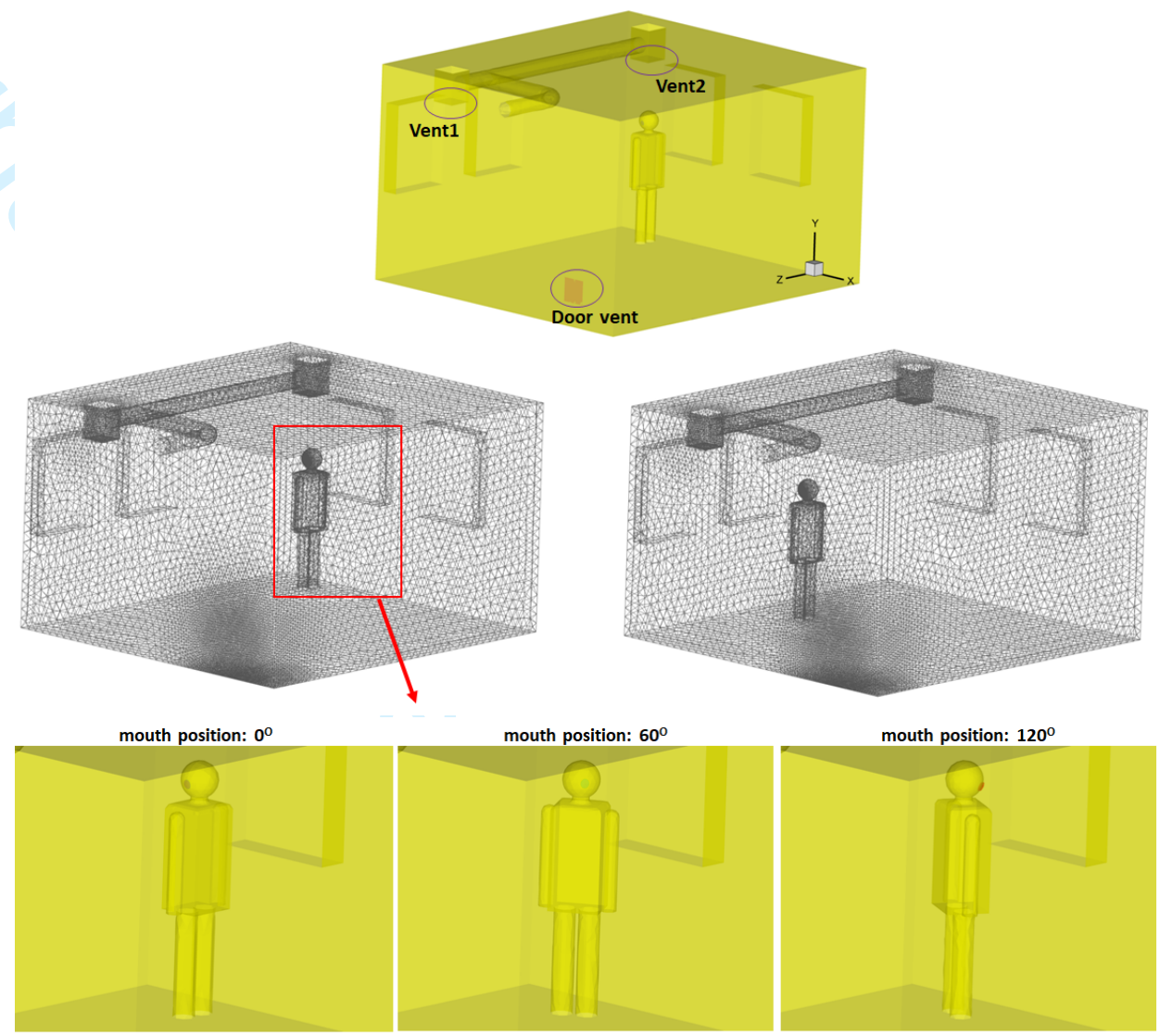

mouth position: $180^{\circ}$

mouth position: $240^{\circ}$

mouth position: $300^{\circ}$
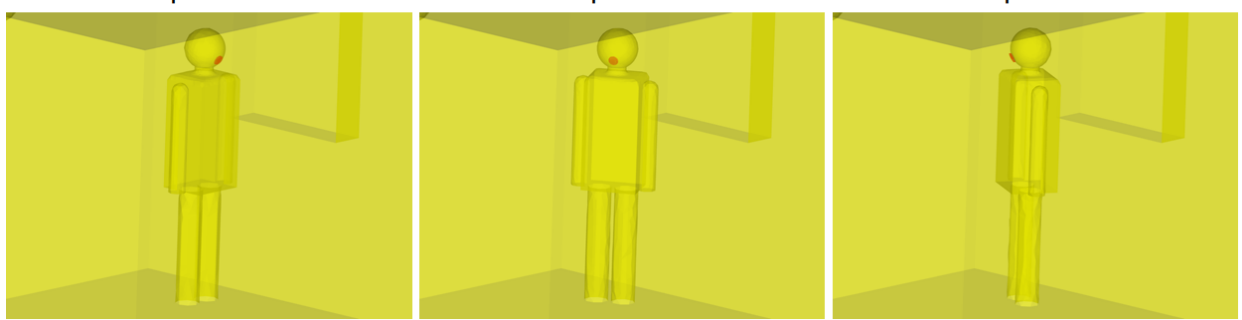

Figure 1: Schematic of model room and corresponding meshes with human standing positions

\begin{tabular}{|c|c|c|l|}
\hline $\begin{array}{c}\text { Meshes } \\
\text { (mouth positions) }\end{array}$ & Elements & Nodes & $\begin{array}{l}\text { Degree of freedom } \\
\text { (velocity, pressure, } \\
\text { turbulent eddy viscosity) }\end{array}$ \\
\hline 0-degree & 1031440 & 176625 & 883125 \\
\hline 60-degree & 1021471 & 175035 & 875175 \\
\hline 120-degree & 1021846 & 175085 & 875425 \\
\hline 180-degree & 1029153 & 176252 & 881260 \\
\hline 240-degree & 1064779 & 182161 & 910805 \\
\hline 300-degree & 1022857 & 175289 & 876445 \\
\hline
\end{tabular}

Table 1: Mesh characteristic parameters, human under vent2 
Reynolds number (Re)

\begin{tabular}{|c|c|c|c|c|c|c|c|c|c|}
\hline Vent1 & 10000 & 20000 & 30000 & 20000 & 40000 & 60000 & 0 & 0 & 0 \\
\hline Vent2 & 10000 & 20000 & 30000 & 0 & 0 & 0 & 20000 & 40000 & 60000 \\
\hline
\end{tabular}

Table 2: Reynolds numbers (Re) for different air vent diffusers

With the reference velocity of $1 \mathrm{~m} / \mathrm{s}$, a non-dimensional diffuser inlet vertical air velocity of unity is imposed. Air flow from mouth is assumed to be exhaled at a velocity inlet boundary condition $2.0 \mathrm{~m} / \mathrm{s}$ for a mouth inlet area of $0.0028 \mathrm{~m}^{2}$. No slip conditions are applied on walls and windows of the room. For the Spalart-Allmaras (SA) model the scalar variable $\hat{\nu}$ is prescribed equal to 1.0 at the inlet and zero on the solid walls. First, the steady-state solution is obtained for the airflow without particles with the convergence criterion to steady state of $10^{-6}$ tolerance value. We assumed that during a single normal breathing around $\mathrm{N}=750$ particles at the person's mouth location are released into the room.

For the ML model, a dataset containing 108 samples/numerical solution for different configurations are generated. Here, the effect of opening both vents with three different Reynolds numbers of $\mathrm{Re}=10000,20000$, and 30000 are considered. Additionally, we also considered the case in which one of the vents is closed and the flow in the other one is doubled (ie, $R e=20000$, 40000, 60000) (see Table 2). For the machine learning (see Section 4), the dataset is split randomly into train/test sets following a $80: 20$ ratio $(80 \%$ of the data for training and $20 \%$ of the data for testing). This database is then used to train the machine learning algorithms. The trained algorithm is then used to predict in time for 1800 seconds (30 minutes), the total number of particles in the air, the total number of particles left the room air through the vent door, the total number of particles attached to the wall, and the particles stuck in the air at locations with zero-velocity. In addition, Gated Recurrent Units (GRUs) are used to speed up the training time and to accelerate the ML workloads.

\section{Neural network architecture}

In this work, a hybrid neural networks comprising of Gated Recurrent Units (GRU) and Multi-Layer Perceptions (MLP) are used to predict the number of particles movement released from normal breathing. The GRU offers a very comparable accuracy to the more widely used Long ShortTerm Memory (LSTM), while incurring a shorter training time [43]. The GRU neural networks [26, 27, 44] is an extended and improved version of the Recurrent Neural Network (RNN) [45, 46] which are designed to work 
with the sequential data architecture and are capable of handling long-term dependencies. A RNN unit takes input from the previous step $\left(c^{<t-1>}\right)$ and current input $\left(x^{<t>}\right)$. The cell state $\left(c^{<t>}\right)$ at the current time is then given by.

$$
c^{<t>}=g\left(W_{c}\left[c^{<t-1>}, x^{<t>}\right]+b_{c}\right)
$$

where $W_{c}$ are weights, $b_{c}$ are biases, or trainable parameters, and $g$ is the activation function. RNN's face short-term memory problem and cannot process very long sequences. It is caused due to vanishing gradient problem. As RNN processes more time steps it suffers from vanishing gradient making them unable to learn long-term dependencies efficiently [47, 48].

Gated Recurrent Units (GRU) is able to process even the longest sequence data without vanishing of the gradient. GRU's are created as the solution to short-term memory. They have internal mechanisms called gates that can regulate the flow of information. As shown in Figure 2, GRU has a complex recurrent structure in a single unit, which is chronologically connected in time. GRU has two gates, reset gate $\left(\Gamma_{r}\right)$ and update gate $\left(\Gamma_{u}\right)$. The reset gates are used to decide how much past knowledge are irrelevant later in the future to drop and update gates and decide what knowledge to be added to the cell state. Each gate has its own weights and biases. The relationship between the input and the output of GRU may be defined by a set of the following equations:

$$
\begin{array}{r}
\hat{c}^{<t>}=\tanh \left(W_{c}\left[\Gamma_{r} * c^{<t-1>}, x^{<t>}\right]+b_{c}\right) \\
\Gamma_{r}=\sigma\left(W_{r}\left[c^{<t-1>}, x^{<t>}\right]+b_{r}\right) \\
\Gamma_{u}=\sigma\left(W_{u}\left[c^{<t-1>}, x^{<t>}\right]+b_{u}\right) \\
c^{<t>}=\Gamma_{u} * \hat{c}^{<t>}+\left(1-\Gamma_{u}\right) * c^{<t-1>}
\end{array}
$$

where $W_{c}, W_{r}$, and $W_{u}$ are the parameter matrices (weights) and $b_{c}, b_{r}$, and $b_{u}$ are bias vectors. $\sigma(x)=\frac{1}{1+\exp (-x)}$ is the element-wise logistic sigmoid function. The corresponding diagram for equations (16) is displayed in Figure 2 .

The main part of the Artificial Neural Networks methodology is the learning or training process in which the errors determined at the output layer are successively reduced by adjusting the weights and biases throughout the network. The back-propagation algorithm changes the weights towards a lower error at the end. The network weights and biases of Neural Networks (NNs) are tuned based on data using the adaptive moment estimation (Adam) [49] algorithm. Adam method is one of the most popular gradient-based optimization algorithms for optimizing neural networks and is computationally efficient, has little memory requirement, and is well suited for problems that are large in terms of data/parameters [49]. In this 


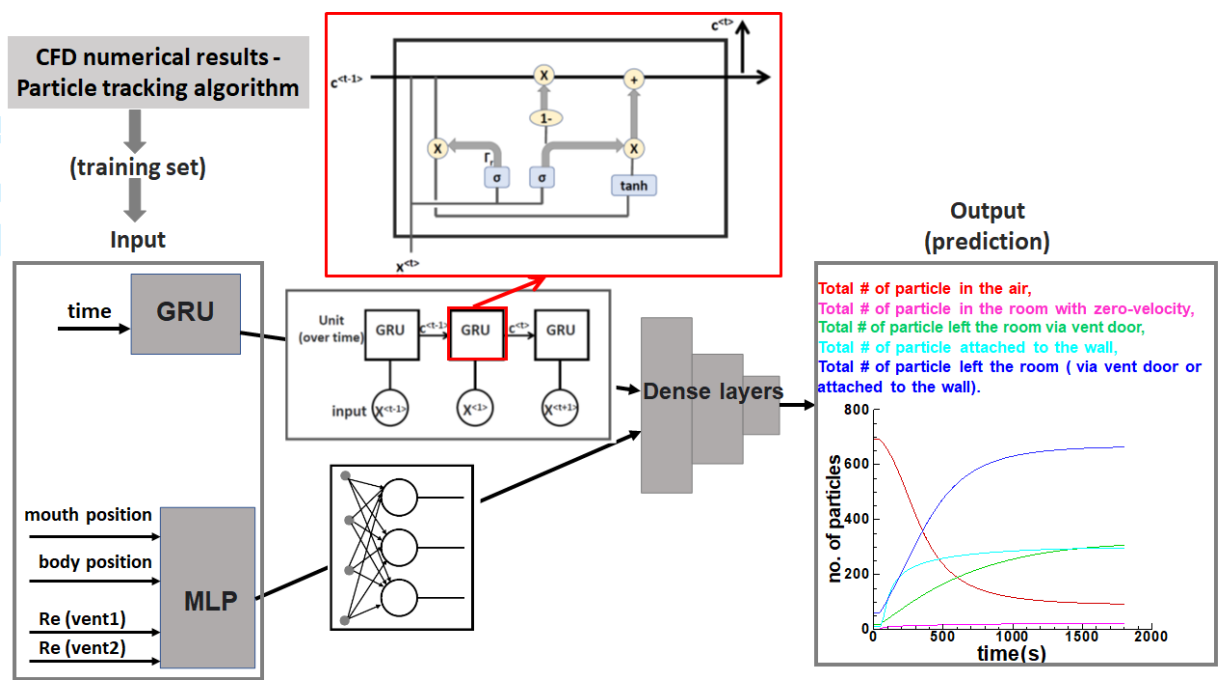

Figure 2: The diagram of the hybrid neural networks comprising of Gated Recurrent Units (GRU) and Multi-Layer Perceptions (MLP), input and output data

work, default setting of hyper-parameters of Adam optimization algorithm are used. For more details on Adam optimizer see [49, 50].

The input to Neural Networks are inlet vent conditions, location of a person in the room with different mouth positions and particle dispersion and deposition. The output of the NN is the total number of particles in the air, the total number of particles left the room through the outlet vent door, the total number of particles attached to the wall, and the particles stuck in the air at locations with zero-velocity. The detailed network configuration and the parameters, the input to Neural Networks, and the output of the NN used in this work are shown Figure 2 and in Table 3. In order to verify the prediction accuracy performance of the proposed model for the continuous-time associated to our airborne movement problem, this paper uses the mean square error (MSE) as the model criterion, ie,

$$
E_{M S E}=\frac{1}{n} \sum_{i=1}^{n}\left(x_{i}-\hat{x}_{i}\right)^{2}
$$

where $\hat{x}_{i}$ is the prediction value and $n$ is the number of sample points in the test data set.

\section{Results and Discussion}

In the present work we analysed three main factors affecting the particle transport: air flow ventilation rates, human standing positions, and human location in the room. In the proposed settings, variations in the ventilation 
Table 3: ANN parameters - airborne movement problem

\begin{tabular}{|l|c|}
\hline RNN cell & Gated Recurrent Unit \\
Number of hidden GRU layers & $2-4$ \\
Number of units within hidden- & 2 or 4 \\
GRU layers & \\
Number of inputs & 5 \\
Number of dense layers & 2 : with 11 and 8 neurons \\
Number of fully connected layers & 1 (output layer, no. of particles in time) \\
Dense activation layers & Tanh (hyperbolic tangent) \\
Output activation layer & Sigmoid function \\
loss function & Mean squared error \\
Number of epochs & 1000 \\
Validation-split & 0.2 \\
Optimiser & Adam (default hyper-parameters) \\
\hline
\end{tabular}

air flow (ie, Re=10000-60000), human standing positions (0, 60, 120, 180, 240 , and 300 degrees), and human location in the room significantly modified the level of air flow and airborne infections response.

\subsection{Effects of ventilation rates}

The role of ventilation rates in airborne infections are shown in Figure 3 for three different vents scenarios where either both vents are open or one of the vents is closed and the Reynolds number on the other vent is doubled. The general trends demonstrate how the infection can be persistently carried by the airflow in the room from one point to another depending on the pattern of the airflow, location of ventilators, and location of the human. In Figure 3, steady state visual representation of airflow trajectories (stream wise, top) and contour plot (middle) colored to velocity magnitude is presented for $R e=20000$ (both vents open), $R e=40000$ (vent1: open, vent2: closed), and $\operatorname{Re}=40000$ (vent1: closed, vent2: open) with the human standing position of 0 degree (see Figure 1). Additionally, in Figure 3 (bottom), the distribution of particles released from normal breathing is displayed in time for 1800 seconds (30 minutes). The red line indicates the total number of particles in the air. Blue line shows the total number of particles left the room air through the vent door [green] or attached to the wall [cian]. The particles stuck in the air at locations with zero-velocity are shown in magenta color. It is clearly apparent that when vent 1 is closed and vent 2 is open (Figure 3. f), the total number of particles left the room air $(\mathrm{O}(400)$, $55 \%$ of the particles, blue line) is less than the case when vent 1 is open and vent2 is closed $(\mathrm{O}(660)$, almost $90 \%$ of the particles, (Figure 3b, e)). This is due to closing the air vent where some particles remain under vent1 and not moving. Furthermore, when both vents are open (Figure 3a, d), the total number of the particles without interacting with any surfaces $(55 \%$, 

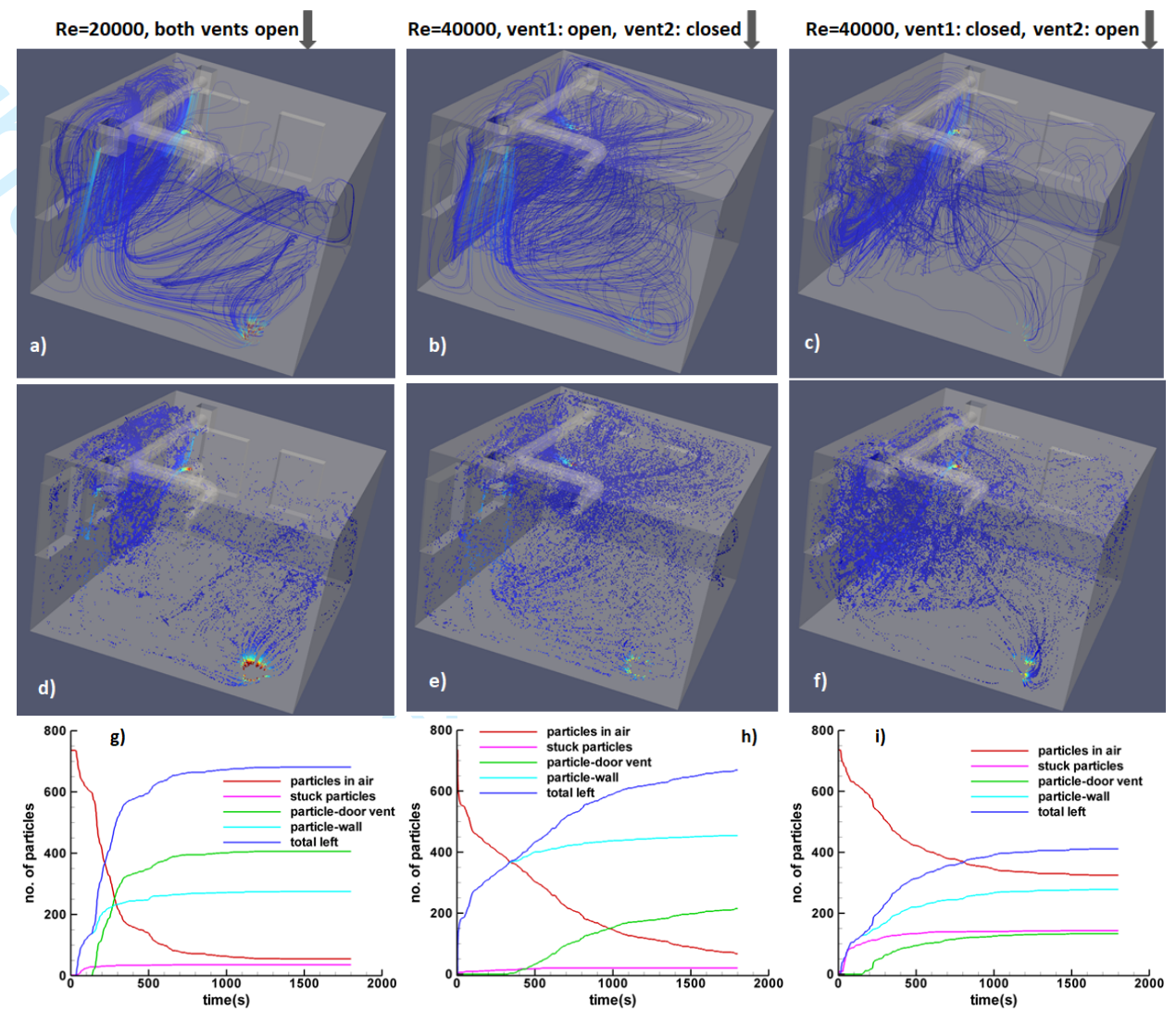

Figure 3: Steady state visual representation of airflow trajectories in active office colored to velocity magnitude (a-f), Distribution of particles released from normal breathing (g-l) in time, human under vent 2 , mouth position: 0 degee, $R e=20000,40000$

Figure 3r) left the room after 30 minutes (green line) are greater than the cases when one of the vents is open (around $30 \%$ and $16 \%$, Figures $3 \mathrm{~h}$, i, green lines). In general, when both vents are open, the particles in air (red line) move rapidly comparing with the cases with only one vent open.

Next, we compare the performance of the hybrid neural network prediction model described in Section 4 with the the numerical results obtained using the CFD model. Figure 5 shows the model loss for the training dataset for Gated Recurrent Unit(GRU) with different level of layers and neurons. Figure 4 shows the CFD-data against the machine learning predictions with three GRU model variants, GRU(4-2), 2 layers with 4 and 2 units, GRU(42-2), 3 GRU layers with 4, 2 and 2 units, and GRU(4-2-2-2), 4 layers with 4, 2, 2 and 2 units, respectively. In Figure 4 , the total number of particles in the air, the particles stuck in the air at locations with zero-velocity, and the total number of particles left the room air through the vent door are captured in 1800 seconds (30) minutes. In this case the values of Reynolds number are taken to be $\operatorname{Re}=40000$ (both vents are open, Figure 4 a) and 

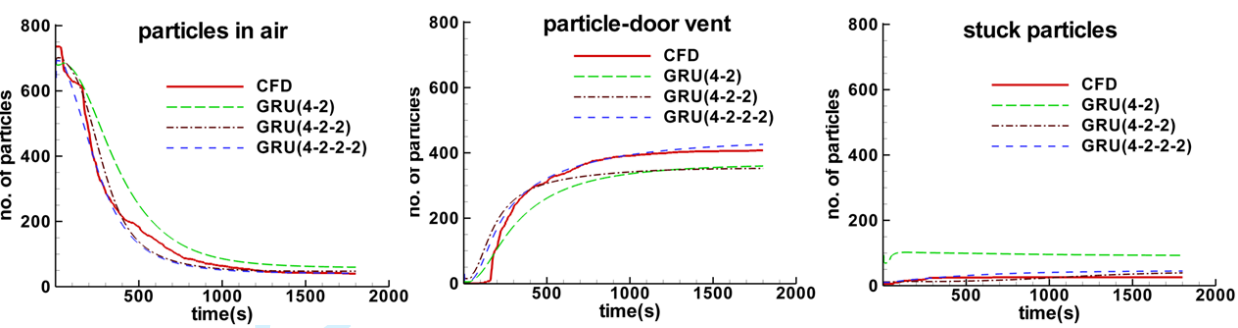

(a)

Re=50000, Vent1 closed, Vent 2 open
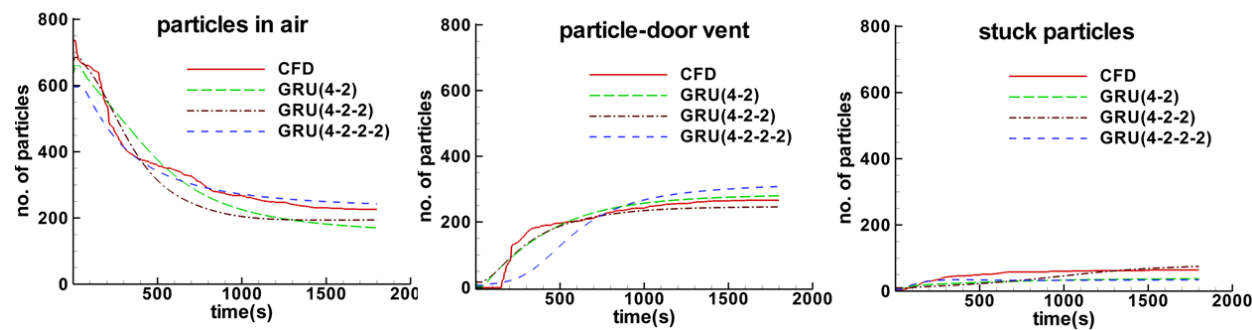

(b)

Figure 4: Distribution of particles released from normal breathing in time, human under vent 2 , mouth position: 0 degree, a) $R e=40000$, both vents open b) $R e=50000$, vent 1 closed, vent2 open, CFD vs Gated Recurrent Unit(GRU) with different level of layers and units

Re $=50000$ (vent1 closed, vent2 open, Figure 4b). Note that, both Reynolds number values are outside the training data range. As shown in Figures $4 \mathrm{a}$ and $4 \mathrm{~b}$, the CFD results (red lines) are captured in a tight window provided by the range of GRUs neural networks (GRU(4-2-2), GRU(4-2), and GRU(4-2)). Here, GRU(4-2-2-2) with more number of trainable parameters presents a closer prediction to CFD findings when compared to its counterpart GRU(4-2).

In addition, model loss for the training dataset for GRU(4-2), GRU(42-2), and GRU(4-2-2-2) are shown in Figure 5. The loss values predicted by the neural network for trained data are around $1.5 \%$ for almost all GRUs, after an initial stabilisation period. Furthermore, very little differences in training loss are observed between the three GRU neural networks. Additionally, the training loss is consistent with increasing number of epochs. This leads to model stability and non overfitting, indicating desirable model performance. 


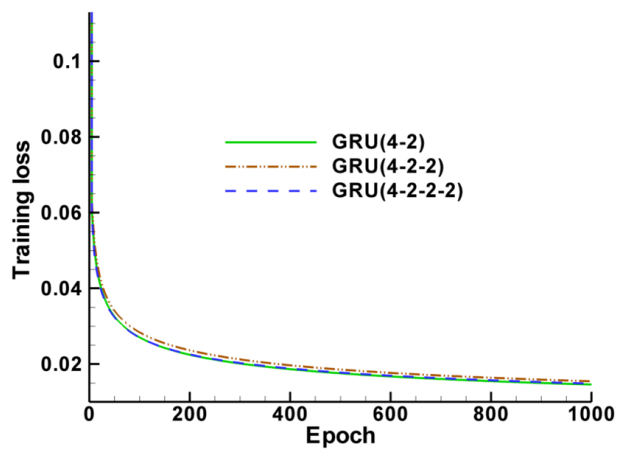

Figure 5: Model loss for the training dataset for Gated Recurrent Unit(GRU) with different level of layers and neurons

\subsection{Effects of human standing position}

The overall impact of human position on air flow trajectories and particle distribution for selected three different human standing positions of 0, 240, and 300 degrees is presented in Figure 6. In this case, Reynolds number is taken to be $\mathrm{Re}=30000$ where both vents are open. In Figure 6c and its counterpart GRUNN-prediction plot in Figure 6f, one may note the rapid decline in number of particles in air (red line) and at the same time rapid increase in the number of particles left the room (blue line). This is due to orientation and mouth position of 300 degree, where the human standing position is in a direction toward the vent 1 and this causes the particles to leave the room much faster when comparing to other cases with human orientation in the room. In this case (mouth position: 300), almost $97 \%$ of the total particles (Figure 6;, blue line) left the room after around 8 minutes (500 seconds). Yet, The total number of particles left the room at the same time ( 8 minutes) with human standing positions of 0 , and 240 degrees are approximately $66 \%$, and $90 \%$, respectively ((Figures $6 \mathrm{~d}, \mathrm{e})$. Moreover, the number of particles attached to the wall [cian] is almost $16 \%$ of the total released particles for the case of 300 degree mouth position after 30 minutes (1800 second), (Figure 6F). The number of particles attached to the wall is increased to around $38 \%$, and $40 \%$ of the total particles, for 240 , and 0 degree mouth position cases, respectively (Figures 6e, d). Furthermore, the maximum number of particles remain in suspension in the system after 30 minutes (1800 seconds) are around 17 particles (4\% of the total particles) for the case of 0 degree mouth position (Figure 6 $\mathrm{d}$ ).

\subsection{Effects of human location in room}

In this section, prediction under two different human locations (human under vent 2 and human in the middle of the room between two vents) are considered, with the purpose of investigating the effects of movement and maintaining social distancing from the infectious person in the room and 

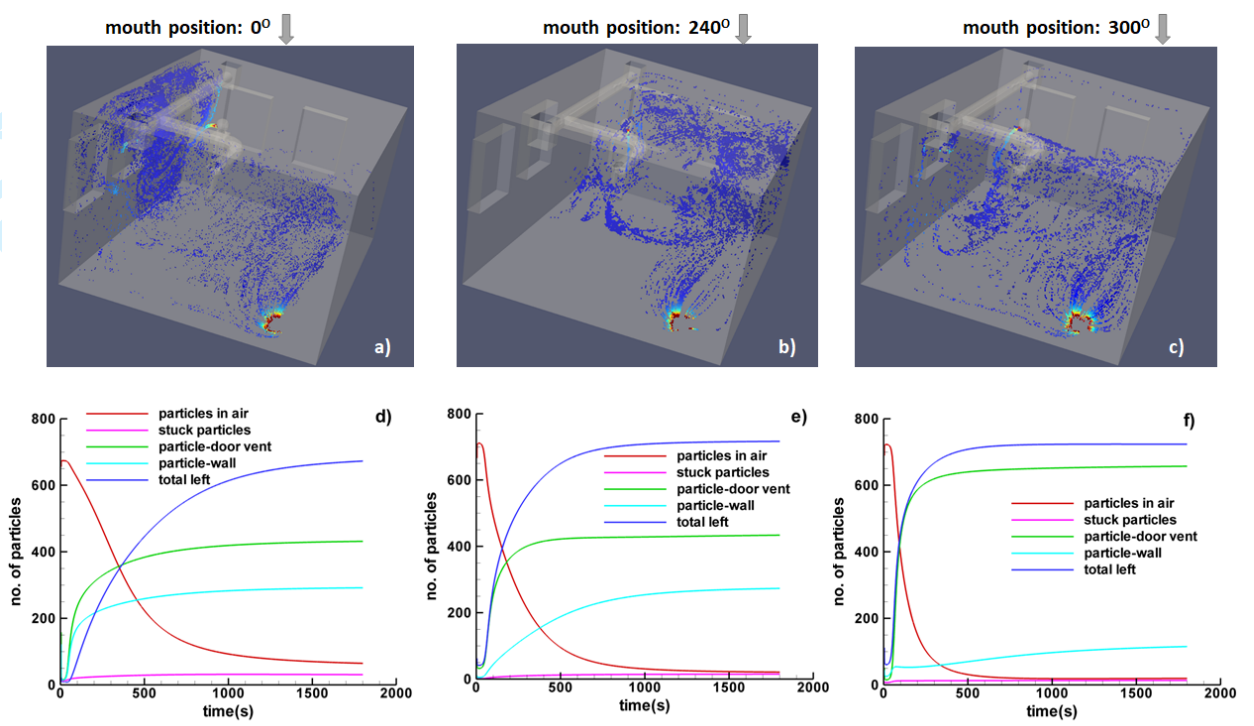

Figure 6: Steady state visual representation of airflow trajectories in active office colored to velocity magnitude (a-c), human under vent $2, \mathrm{Re}=30000$, prediction of particles movement released from normal breathing (d-f) in time, GRU(4-2-2-2)

predicting of particle movement released from normal breathing. In Figure $7 \mathrm{a}-\mathrm{d}$, steady state visual representation of airflow trajectories is presented for $R e=30000$ with the human standing position of 120 , and 180 degrees. In addition, for the same setting, the Neural network for prediction of number of particles movement in time is shown in Figure 7 p-h. As expected, the particles spread more through the space when the human is in the middle of the room between the vents (Figure $7 \mathrm{k}, \mathrm{d}$ ) compared to the case when the human is at the corner of the room under vent2 (Figure $7 a, b)$. Considering the orientation and mouth position of 180 degree with human in the middle of the room (Figure 7h), a sharp drop in particle movement in air (red line) is evident when comparing to its counterpart in Figure $7 \mathrm{f}$ with human at the corner of the room under vent2. Moreover, almost $40 \%$ of the particles left the room air directly through the vent door (Figure 7p,f, green lines) whilst this increases to $68 \%$ in the case with human in the middle of the room between vents (Figure $7 \mathrm{~g}, \mathrm{~h}$ ). Additionally, Figure $7 \mathrm{~h}$ with human in the middle between vents and with mouth position of 180 degree reports lowest number of particles $(13 \%)$ attached to the wall (cian line).

Finally, Figure 8 demonstrates that the Gated Recurrent Units Neural Network (GRU-NN) is capable of predicting the aerosol movement under various ventilation conditions with different human mouth position. Note that, all the inputs for the GRU-NN prediction model in Figure $8 \mathrm{a}-\mathrm{f}$ are taken to be outside the training data range. 

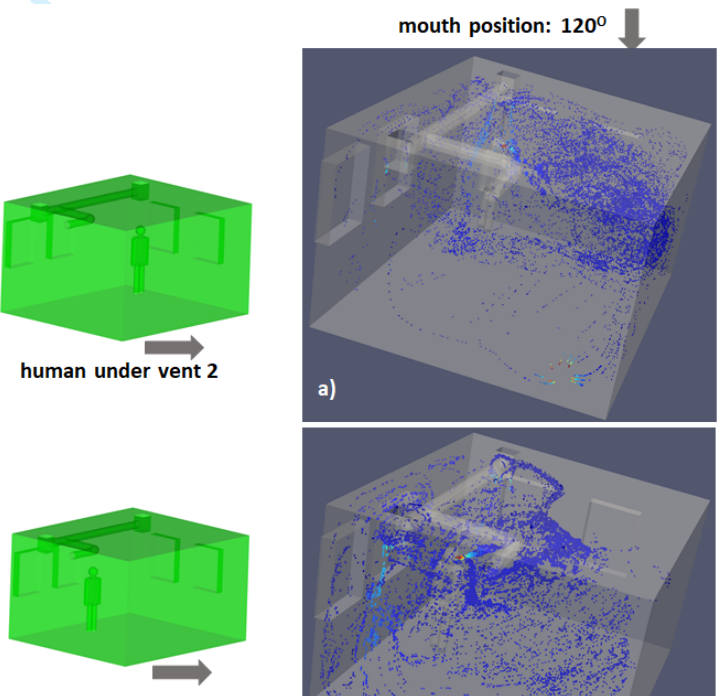

human in the middle between vents
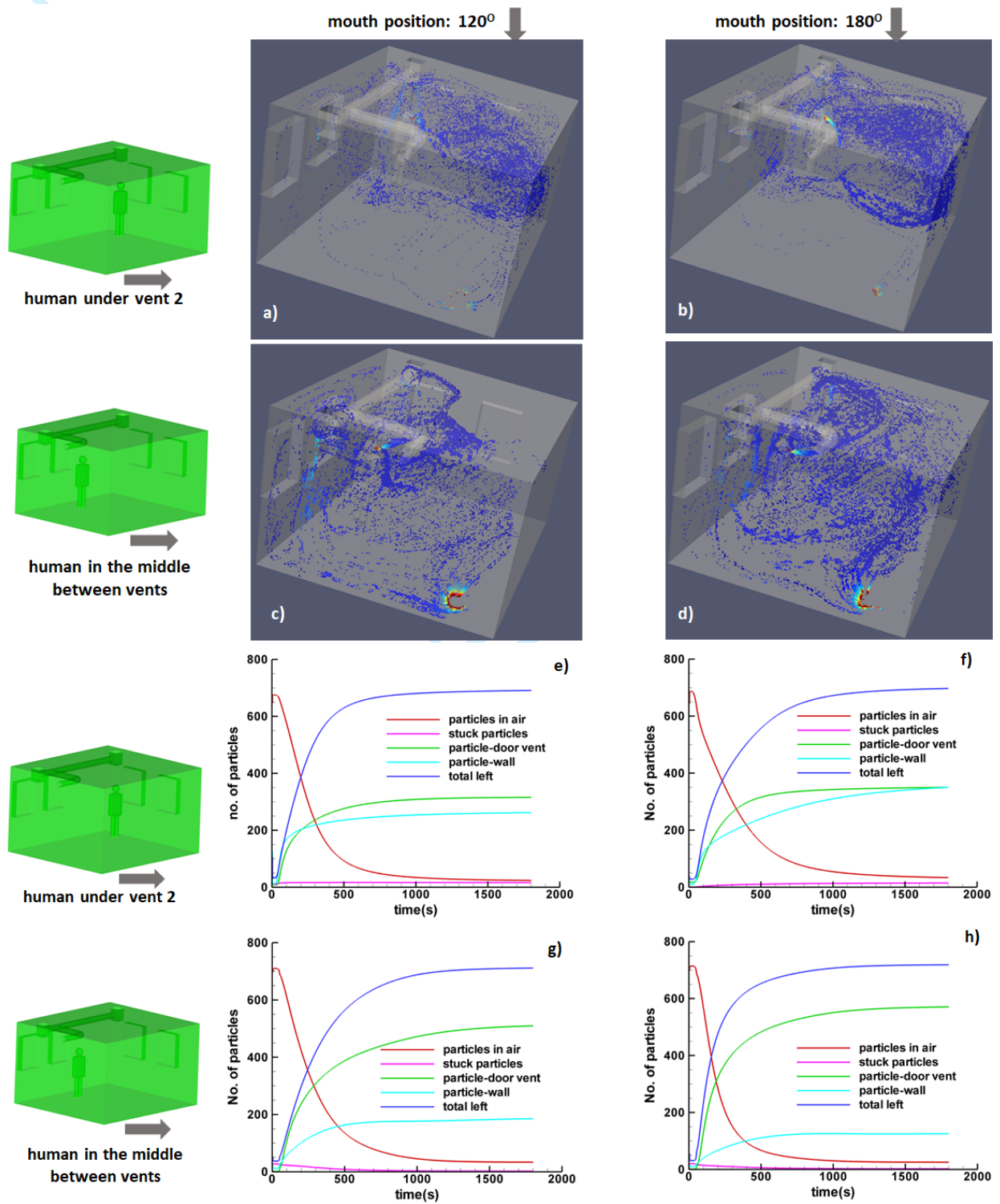

Figure 7: Steady state visual representation of airflow trajectories in active office colored to velocity magnitude, human under vent2 $(a, b)$, human in the middle between vents $(\mathrm{c}, \mathrm{d}), \mathrm{Re}=30000$, prediction of particles movement released from normal breathing (d-f) in time, GRU(4-2-2-2), human under vent2 (e,f), human in the middle between vents (g,h) 

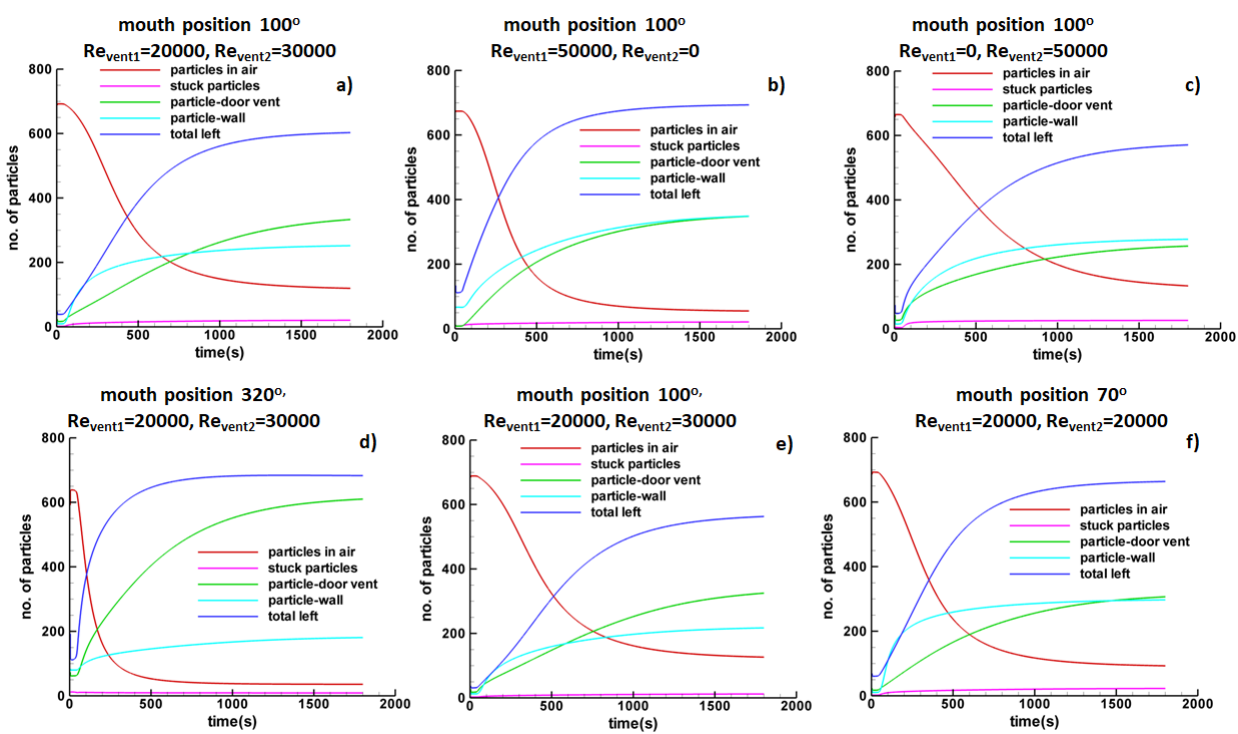

Figure 8: Prediction of particles movement released from normal breathing (a-f) in time with GRU(4-2-2-2), Various Re and human mouth positions

\section{Conclusions}

Through this work a predictive method for the accurate capture of infectious particle behaviours, originated during normal breathing of a human, is established. The methodology is based on a Gated Recurrent Units Neural Network (GRUs-NN) model, which is capable of handling long-term dependencies. High-fidelity prediction of indoor air flow is obtained by means of an in-house parallel CFD solver which employs a one equation Spalrat-Allmaras (SA) turbulence model. Several flow scenarios regarding the operation of inlet vents, location of a person in an active office with different human standing positions and particle dispersion and deposition in the enclosed environment are considered. The airflow pattern shows how the particles can be carried by the airflow within active office. The recorded airflow pattern in the active office can be very complex, depending on the location of air ventilations and ventilation rates, as well as on the position of particles. Movement of people within the room can further complicate the resulting fluid dynamics. Through the proposed cases we showed that the Gated Recurrent Units Neural Network model can provide predictions in time which are in good agreement with the CFD numerical results. This study paves the way for the development of efficient and reliable tools for predicting virus airborne movement under different ventilation conditions and different human positions within an indoor environments, potentially leading to new design. 


\section{Acknowledgements}

Authors acknowledge the financial support from Ser Cymru III - Tackling Covid 19 fund, Welsh Government Project number 095. The authors are also grateful for helpful discussion with Dr Justin Searle and active office team, SPECIFIC Innovation \& Knowledge Centre, Swansea University, UK.

\section{References}

[1] Michael A Kohanski, L James Lo, and Michael S Waring. Review of indoor aerosol generation, transport, and control in the context of COVID-19. In International forum of allergy \& rhinology, volume 10, pages 1173-1179. Wiley Online Library, 2020.

[2] Neeltje Van Doremalen, Trenton Bushmaker, Dylan H. Morris, Myndi G. Holbrook, Amandine Gamble, Brandi N. Williamson, Azaibi Tamin, Jennifer L. Harcourt, Natalie J. Thornburg, Susan I. Gerber, et al. Aerosol and surface stability of SARS-CoV-2 as compared with SARS-CoV-1. New England Journal of Medicine, 382(16):1564-1567, 2020 .

[3] Lidia Morawska and D.K. Milton. It Is Time to Address Airborne Transmission of Coronavirus Disease 2019 (COVID-19). Clinical Infectious Diseases, 71(9):2311-2313, 2020.

[4] Mengjia Tang, Ningling Zhu, Kerry Kinney, and Atila Novoselac. Transport of indoor aerosols to hidden interior spaces. Aerosol Science and Technology, 54(1):94-110, 2020.

[5] Ramon Codina, Christopher Morton, Eugenio Onate, and Orlando Soto. Numerical aerodynamic analysis of large buildings using a finite element model with application to a telescope building. International Journal of Numerical Methods for Heat \& Fluid Flow, 10(6):613-633, 2000 .

[6] Souad Morsli, Mustapha Boussoufi, Amina Sabeur, Mohammed El Ganaoui, and Rachid Bennacer. Small to large scale mixed turbulent convection: buildings application. International Journal of Numerical Methods for Heat \& Fluid Flow, 28(1):188-205, 2018.

[7] Zohir Younsi, Lounes Koufi, and Hassane Naji. Numerical study of the effects of ventilated cavities outlet location on thermal comfort and air quality. International Journal of Numerical Methods for Heat \& Fluid Flow, 29(11):4462-4483, 2019. 
[8] Pedro M de Oliveira, Leo CC Mesquita, Savvas Gkantonas, Andrea Giusti, and Epaminondas Mastorakos. Evolution of spray and aerosol from respiratory releases: theoretical estimates for insight on viral transmission. Proceedings of the Royal Society A, 477(2245):20200584, 2021.

[9] Khaled Talaat and Jinxiang Xi. Computational modeling of aerosol transport, dispersion, and deposition in rhythmically expanding and contracting terminal alveoli. Journal of Aerosol Science, 112:19-33, 2017.

[10] Vinh Van Tran, Duckshin Park, and Young-Chul Lee. Indoor air pollution, related human diseases, and recent trends in the control and improvement of indoor air quality. International Journal of Environmental Research and Public Health, 17(8):2927, 2020.

[11] Ioannis Manisalidis, Elisavet Stavropoulou, Agathangelos Stavropoulos, and Eugenia Bezirtzoglou. Environmental and health impacts of air pollution: A review. Frontiers in public health, 8, 2020.

[12] Luis Alfredo Anchordoqui and Eugene M Chudnovsky. A Physicist View of COVID-19 Airborne Infection through Convective Airflow in Indoor Spaces. SciMedicine Journal, 2:68-72, 2020.

[13] D Fontes, J Reyes, K Ahmed, and M Kinzel. A study of fluid dynamics and human physiology factors driving droplet dispersion from a human sneeze. Physics of Fluids, 32(11):111904, 2020.

[14] Sanjay Kumar and Heow Pueh Lee. The perspective of fluid flow behavior of respiratory droplets and aerosols through the facemasks in context of SARS-CoV-2. Physics of Fluids, 32(11):111301, 2020.

[15] Ville Vuorinen, Mia Aarnio, Mikko Alava, Ville Alopaeus, Nina Atanasova, Mikko Auvinen, Nallannan Balasubramanian, Hadi Bordbar, Panu Erästö, Rafael Grande, et al. Modelling aerosol transport and virus exposure with numerical simulations in relation to SARSCoV-2 transmission by inhalation indoors. Safety Science, 130:104866, 2020 .

[16] Rainald Löhner and Harbir Antil. High fidelity modeling of aerosol pathogen propagation in built environments with moving pedestrians. International Journal for Numerical Methods in Biomedical Engineering, 37(3):e3428, 2021.

[17] Rainald Löhner, Harbir Antil, Sergio Idelsohn, and Eugenio Oñate. Detailed simulation of viral propagation in the built environment. Computational Mechanics, 66(5):1093-1107, 2020. 
[18] Mohamed Abuhegazy, Khaled Talaat, Osman Anderoglu, and Svetlana V Poroseva. Numerical investigation of aerosol transport in a classroom with relevance to COVID-19. Physics of Fluids, 32(10):103311, 2020 .

[19] Zechariah Lau, Katerina Kaouri, and Ian M Griffiths. Modelling Airborne Transmission of COVID-19 in Indoor Spaces Using an AdvectionDiffusion-Reaction Equation. arXiv preprint arXiv:2012.12267, 2020.

[20] Z Lau, IM Griffiths, A English, and K Kaouri. Predicting the Spatially Varying Infection Risk in Indoor Spaces Using an Efficient Airborne Transmission Model. 2021.

[21] Yiping Li, Gabriel M Leung, JW Tang, Xiaozhan Yang, CY Chao, John Zhang Lin, JW Lu, Per Væggemose Nielsen, Jianlei Niu, H Qian, et al. Role of ventilation in airborne transmission of infectious agents in the built environment-a multidisciplinary systematic review. Indoor air, 17(1):2-18, 2007.

[22] Shengwei Zhu, Sara Jenkins, Kofi Addo, Mohammad Heidarinejad, Sebastian A Romo, Avery Layne, Joshua Ehizibolo, Daniel Dalgo, Nicholas W Mattise, Filbert Hong, et al. Ventilation and laboratory confirmed acute respiratory infection (ARI) rates in college residence halls in College Park, Maryland. Environment international, 137:105537, 2020.

[23] Hua Qian and Xiaohong Zheng. Ventilation control for airborne transmission of human exhaled bio-aerosols in buildings. Journal of thoracic disease, 10(Suppl 19):S2295, 2018.

[24] Tom Lipinski, Darem Ahmad, Nicolas Serey, and Hussam Jouhara. Review of ventilation strategies to reduce the risk of disease transmission in high occupancy buildings. International Journal of Thermofluids, page $100045,2020$.

[25] Yi Luo, Liehui Zhang, Yin Feng, and Yulong Zhao. Three-Dimensional Streamline Tracing Method over Tetrahedral Domains. Energies, $13(22): 6027,2020$.

[26] Kyunghyun Cho, Bart Van Merriënboer, Dzmitry Bahdanau, and Yoshua Bengio. On the properties of neural machine translation: Encoder-decoder approaches. arXiv preprint arXiv:1409.1259, 2014.

[27] Junyoung Chung, Caglar Gulcehre, K Cho, and Y Bengio. Empirical evaluation of gated recurrent neural networks on sequence modeling. arXiv 2014. arXiv preprint arXiv:1412.3555, 2014. 
[28] Sumit Das, Aritra Dey, Akash Pal, and Nabamita Roy. Applications of artificial intelligence in machine learning: review and prospect. International Journal of Computer Applications, 115(9), 2015.

[29] Devanshi Dhall, Ravinder Kaur, and Mamta Juneja. Machine learning: a review of the algorithms and its applications. Proceedings of ICRIC 2019, pages 47-63, 2020.

[30] Yoshua Bengio. Learning deep architectures for AI. Foundations and trends(R) in Machine Learning, 2(1):1-127, 2009.

[31] Capuano, German and Rimoli, Julian J. Smart finite elements: A novel machine learning application. Computer Methods in Applied Mechanics and Engineering, 345:363-381, 2019.

[32] Tamaddon-Jahromi, Hamid Reza and Chakshu, Neeraj Kavan and Sazonov, Igor and Evans, Llion M and Thomas, Hywel and Nithiarasu, Perumal. Data-driven inverse modelling through neural network (deep learning) and computational heat transfer. Computer Methods in Applied Mechanics and Engineering, 369:113217, 2020.

[33] Rainald Löhner, Harbir Antil, Hamid Tamaddon-Jahromi, Neeraj Kavan Chakshu, and Perumal Nithiarasu. Deep learning or interpolation for inverse modelling of heat and fluid flow problems? International Journal of Numerical Methods for Heat \& Fluid Flow, 2021.

[34] J. Clarke. Active Classroom Case Study. Technical report, Architectural Design Manager, SPECIFIC, Version 2.0, 2020.

[35] Philippe Spalart and Steven Allmaras. A one-equation turbulence model for aerodynamic flows. In 30th aerospace sciences meeting and exhibit, page 439, 1992.

[36] Steven R Allmaras and Forrester T Johnson. Modifications and clarifications for the implementation of the spalart-allmaras turbulence model. In Seventh international conference on computational fluid $d y$ namics (ICCFD'), pages 1-11, 2012.

[37] P. Nithiarasu and C. B. Liu. An artificial compressibility based characteristic based split (cbs) scheme for steady and unsteady turbulent incompressible flows. Computer Methods in Applied Mechanics and Engineering, 195(23-24):2961-2982, 2006.

[38] HamidReza Tamaddon Jahromi, Samuel Rolland, Jason Jones, Alberto Coccarelli, Igor Sazonov, Chris Kershaw, Chedly Tizaoui, Peter Holliman, David Worsley, Hywel Thomas, et al. Modelling ozone disinfection process for creating COVID-19 secure spaces. International Journal of Numerical Methods for Heat \& Fluid Flow, 2021. 
[39] Zienkiewicz, OC and Taylor, RL and Nithiarasu, P. Introduction to the equations of fluid dynamics and the finite element approximation. The Finite Element Method for Fluid Dynamics. 7th ed. Oxford: Elsevier Butterworth-Heinemann, pages 1-29, 2014.

[40] P Nithiarasu, JS Mathur, NP Weatherill, and K Morgan. Threedimensional incompressible flow calculations using the characteristic based split (CBS) scheme. International Journal for Numerical Methods in Fluids, 44(11):1207-1229, 2004.

[41] Codina, Ramon and Coppola-Owen, $\mathrm{H}$ and Nithiarasu, P and Liu, CB. Numerical comparison of CBS and SGS as stabilization techniques for the incompressible Navier-Stokes equations. International Journal for Numerical Methods in Engineering, 66(10):1672-1689, 2006.

[42] Nithiarasu, P and Liu, C-B and Massarotti, N. Laminar and turbulent flow calculations through a model human upper airway using unstructured meshes. Communications in Numerical Methods in Engineering, 23(12):1057-1069, 2007.

[43] Rajib Rana. Gated recurrent unit (GRU) for emotion classification from noisy speech. arXiv preprint arXiv:1612.07778, 2016.

[44] Gail Weiss, Yoav Goldberg, and Eran Yahav. On the practical computational power of finite precision rnns for language recognition. arXiv preprint arXiv:1805.04908, 2018.

[45] Rumelhart, David E and Hinton, Geoffrey E and Williams, Ronald J. Learning representations by back-propagating errors. nature, 323(6088):533-536, 1986.

[46] Erdenebayar Urtnasan, Jong-Uk Park, and Kyoung-Joung Lee. Automatic detection of sleep-disordered breathing events using recurrent neural networks from an electrocardiogram signal. Neural computing and applications, 32(9):4733-4742, 2020.

[47] Lechner, M and Hasani, R. Learning Long-Term Dependencies in Irregularly-Sampled Time Series. arXiv 2020. arXiv preprint arXiv:2006.04418.

[48] Ribeiro, Antônio H and Tiels, Koen and Aguirre, Luis A and Schön, Thomas. Beyond exploding and vanishing gradients: analysing RNN training using attractors and smoothness. In International Conference on Artificial Intelligence and Statistics, pages 2370-2380. PMLR, 2020.

[49] Adam D.P. Kingma, J. Ba. A method for stochastic optimization. Anon. International Conferenceon Learning Representations. SanDego: ICLR, 2015. 
[50] Sashank J Reddi, Satyen Kale, and Sanjiv Kumar. On the convergence of adam and beyond. arXiv preprint arXiv:1904.09237, 2019.

1

2

3

4

5

6

7

8

9

10

11

12

13

14

15

16

17

18

19

20

21

22

23

24

25

26

27

28

29

30

31

32

33

34

35

36

37

38

39

40

41

42

43

44

45

46

47

48

49

50

51

52

53

54

55 


\section{Predicting the airborne microbial transmission via human breath particles using a Gated Recurrent Units neural network}

\section{Abstract}

\section{Purpose}

The main purpose of this paper is to devise a tool, based on Computational Fluid Dynamics (CFD) and Machine Learning (ML), for the assessment of potential airborne microbial transmission in enclosed spaces. A Gated Recurrent Units Neural Network (GRU-NN) is presented to learn and predict the behaviour of droplets expelled through breaths via particle tracking datasets.

\section{Design/methodology/approach}

A computational methodology is used for investigating how infectious particles originated in one location are transported by air and spread throughout a room. High-fidelity prediction of indoor air flow is obtained by means of an in-house parallel CFD solver which employs a one equation Spalrat-Allmaras (SA) turbulence model. Several flow scenarios are considered by varying different ventilation conditions and source locations. The CFD model is used for computing the trajectories of the particles emitted human breath. The numerical results are used to the ML training.

\section{Finding}

In this work, it is shown that the developed ML model, based on the Gated Recurrent Units Neural Network (GRU-NN), can accurately predict the airborne particle movement across an indoor environment for different vent operation conditions and source locations. The numerical results in the paper prove that the presented methodology is able to provide accurate predictions of the time evolution of particle distribution at different locations of the enclosed space.

\section{Originality/value}

This study paves the way for the development of efficient and reliable tools for predicting virus airborne movement under different ventilation conditions and different human positions within an indoor environments, potentially leading to new design. A parametric study is carried out to evaluate 
the impact of system settings on the time variation particles emitted human breath within the space considered.

Keywords: COVID-19 infection, CFD modelling, Spalrat-Allmaras (SA) model, Particle tracking, Inhalation airflow, Recurrent Neural Network, Gated Recurrent Units (GRU)

\section{Introduction}

In indoor environments, the main transmission route of COVID-19 involves the emission of respiratory droplets from the mouth and nose which can remain suspended in the air for several minutes, exposing the surrounding people to high infection risk [1-4]. In this context, different methodologies for characterizing the fluid dynamics patterns within the indoor environment have been proposed [5] 8 . These efforts have also been accompanied by recent research focusing on how pollution and biological agents can spread throughout an enclosed space [9 14. Recently, Vuorinen, et al. [15] modelled physical processes related to aerosol dispersion in air and focused on transmission by inhalation in the context of COVID-19. These authors gave various examples on the transport and dilution of aerosol dimeters of $d \leq 20$ $\mu m$ over distances $O(10 \mathrm{~m})$ in public indoor environments by Monte-Carlo modelling. Furthermore, Löhner et al. [16, 17] studied the characteristics of virus contaminants and the transmission via droplets and aerosols in a narrow corridor with moving pedestrians and in a typical hospital rooms considering a bi-directional coupling, whereby the flow and the motion of the crowd are computed concurrently and with mutual influences. In subsequent work, Abuhegazy et al.[18] investigated aerosol removal and surface deposition in a realistic classroom with nine students and a teacher using computational fluid particle dynamics algorithm implemented by Ansys Fluent. These authors [18] found that a $24 \%-50 \%$ of particles smaller than $15 \mu \mathrm{m}$ exit the system within 15 minutes through the air conditioning system and particles larger than $20 \mu \mathrm{m}$ almost entirely deposit on the ground, desks, and nearby surfaces in the room. Additionally Lau et al. [19, 20] described a model for indoors airborne transmission where the concentration of airborne infectious particles governed by an advection-diffusion-reaction equation. These authors compared the model both with more complex models and with experimental data and found good agreement. Moreover, to address the relevant background, the impact of ventilation on the airflow pattern has been also extensively studied [21 23]. Ventilation plays an important role in reducing the risk of transmission through dilution and removal of the infected particles within the indoor environment [24].

Despite aerosol transport within indoor environments has been extensively studied in the last decades [25], there is a pressing need for the establishment of efficient computational tools for the prediction of transmission 
and infectivity level of airborne viruses (such as COVID-19 virus) throughout enclosed spaces. To the authors' knowledge, ML methods and specifically Gated Recurrent Units (GRU) neural network [26, 27] have not been employed before for this purpose. With all the information available regarding the migration of airborne infectious particles in indoor environments, a robust control tool to capture, store and analyse data using ML algorithms is essential. Machine Learning represents an efficient and accurate approach to find patterns in the most complex and abstract data by proposing alternatives to analysing large volume of data to forward-looking predictive models [28, 29]. ML has attracted strong interest over many years and is a standard tool today in many applied science topics. The main advantage of ML lies in that the computer can achieve the purpose of self-learning and predict the trend through operating algorithms. Because of this feature, the computer can be continuously trained, the training dataset can be increased, and over time more accurate results can be obtained through data accumulation by developing fast and efficient algorithms 30 33].

Through the present study we devised a combined ML and CFD modelling approach for defining the particle distribution associated to airflow patterns within an indoor environment. This includes natural circulation inside enclosed spaces by air-conditioning with several flow scenarios regarding the operation of inlet vents, location of a person in an active office 34 with different human standing positions, analysing the potential of virus spread through the air from an infected person, identification of critical points, and particle dispersion and deposition in the enclosed environment. Moreover, a Gated Recurrent Units Neural Network (GRU-NN) is presented to learn and predict the behaviour of droplets expelled through breaths via particle tracking datasets. This article is organised as follows: Section 2 provides the details on the adopted computational methodology including the particle tracking scheme for modelling infectious particle behaviours. The description of the problem considered is reported in Section 3. The neural network architecture methodology is described in Section 4. This is followed by the Section 5 in which the results are reported. In the last section, the significant findings of the study are summarised.

\section{Mathematical modelling and solution method}

The forced air circulation within an active office may be described by using turbulent incompressible flow equations. These equations and their solution method are briefly summarised in this section.

\subsection{Air flow within the room}

The motion of the fluid throughout the room is described by means of the incompressible Navier-Stokes equations, combined with a turbulence 
model. The mass and momentum conservation equations in dimensional form read:

$$
\begin{aligned}
& \nabla \cdot \boldsymbol{v}=0, \\
& \frac{\partial \boldsymbol{v}}{\partial t}=-(\boldsymbol{v} \cdot \nabla) \boldsymbol{v}-\frac{1}{\rho} \nabla p+\left(\nu+\nu_{T}\right) \nabla^{2} \boldsymbol{v},
\end{aligned}
$$

where $\boldsymbol{v}$ is the velocity vector, $\rho$ is the air density, $p$ is the pressure, $\nu$ is the kinematic viscosity, whilst $\nu_{T}$ is the turbulent eddy viscosity. The space and time distribution of the turbulent eddy viscosity $\nu_{T}$ is obtained by employing the one equation Spalart-Allmaras (SA) model [35]38], which uses several turbulence parameters $\left(c_{b 1}, \sigma, c_{b 2}, k, c_{w 1}, c_{w 2}, c_{w 3}\right.$ and $\left.c_{v 1}\right)$ for describing the transport of the variable $\hat{\nu}=\nu_{T} / f_{v 1}$. The scalar equation is:

$$
\frac{\partial \hat{\nu}}{\partial t}=-\boldsymbol{v} \cdot \nabla \hat{\nu}+c_{b 1} \hat{S} \hat{\nu}+\frac{1}{\sigma}\left[\nabla \cdot((\nu+\hat{\nu}) \nabla \hat{\nu})+c_{b 2}(\nabla \hat{\nu})^{2}\right]-c_{w 1} f_{w}\left[\frac{\hat{\nu}}{y}\right]^{2}
$$

where

$$
\begin{aligned}
& \hat{S}=S+f_{v 2} \frac{\hat{\nu}}{k^{2} y^{2}} \\
& f_{v 2}=1-X /\left(1+X f_{v 1}\right), \\
& f_{v 1}=X^{3} /\left(X^{3}+c_{v 1}^{3}\right), \\
& f_{w}=g\left[\frac{1+c_{w 3}^{6}}{g^{6}+c_{w 3}^{3}}\right]^{1 / 6}, \\
& X=\hat{\nu} / \nu \\
& g=r+c_{w 2}\left(r^{6}-r\right), \\
& r=\frac{\hat{\nu}}{\hat{S} k^{2} y^{2}}
\end{aligned}
$$

in which $S$ is the magnitude of vorticity and $y$ is the near wall distance. The turbulence parameters are set as follows: $c_{b 1}=0.1355, \sigma=2 / 3, c_{b 2}=$ $0.622, k=0.41, c_{w 1}=c_{b 1} / k^{2}+\left(1+c_{b 2}\right) / \sigma, c_{w 2}=0.3, c_{w 3}=2$ and $c_{v 1}=7.1$.

The equations above are solved by using an in-house parallel CFD library based on a established finite-element characteristic-based split (CBS) scheme, which is suitable for problems with unstructured meshes [37, 39]. The velocity and pressure fields within the room are computed in time by solving Equations 1, 2, in conjunction with the SA turbulence model (Equation 3). Here we used the semi-implicit (in time) version of the CBS since it represent a good compromise between efficiency, accuracy and flexibility for external component integration [40]. These features make the scheme ideal for computing incompressible flow in complex geometries. The code is written in Fortran90 and all simulations are carried out using an Open shift Container Platform (OCP) cluster on 40 processors with the OpenMPI. 


\subsection{Particle tracking}

To calculate the trajectory of a particle drifted by the air current in the room, we evaluate the particle pathway in every mesh cell met by the particle during its travel. Since we use a tetrahedral mesh, the calculation of trajectory requires tracing a particle through a tetrahedron. Here, it is necessary to calculate the leaving point in the tetrahedron surface for given entering point alongside the propagation time trough the cell [25]. For this, we use the linear shape functions $\xi_{i}$ associated to the nodes of the tetrahedron. In this way, any point $\boldsymbol{P}$ inside the cell can be determined as a linear combination of the coordinates of the tetrahedron vertices $\boldsymbol{P}_{i}$ :

$$
\boldsymbol{P}=\sum_{i=1}^{4} \xi_{i} \boldsymbol{P}_{i}
$$

where $\sum_{i=1}^{4} \xi_{i}(\boldsymbol{P})=1$.

Here the velocities are computed at cell vertices, and the velocity varies linearly in space over every cell. The velocity at any point $\boldsymbol{P}$ can be expressed through its shape functions

$$
\boldsymbol{v}=\sum_{i=1}^{4} \xi_{i} \boldsymbol{v}_{i}
$$

where $\boldsymbol{v}_{i}$ are velocities at tetrahedron vertices. By solving a system of three linear equations we can represent velocity $\boldsymbol{v}$ at $\boldsymbol{P}$ through coordinates of tetrahedron vertices in the following form

$$
\boldsymbol{v}=\sum_{i=1}^{4} \hat{v}_{i} \boldsymbol{P}_{i}, \quad \sum_{i=1}^{4} \hat{v}_{i}=0 .
$$

The linear shape functions $\xi_{i}$ also can be treated as coordinates in the master element. Then every parameter $\hat{v}_{i}$ in 13 represents a velocity component along $i$ th master element coordinate $\xi_{i}$. This enables calculation of the propagation time of the particle to the plane of every face and finding which face can be reached first, i.e. the face containing the leaving point.

A trajectory in the linearly varying velocity field can be expressed analytically through the exponential function, but to find the leaving point we have to solve numerically an algebraic equation (see [25]). Instead we propose here a fast and accurate predictor-corrector type method. First, we calculate the values of shape functions for the entering point $\boldsymbol{P}^{\text {in }}$ and, employing equation 12 , calculate the velocity vector $\boldsymbol{v}^{\text {in }}$ at it. Considering velocity $\boldsymbol{v}^{\text {in }}$ as the uniform velocity, we calculate the master element velocity components 13 and the predicted leaving point $\boldsymbol{P}_{*}^{\text {out }}$. Second, we calculate the velocity $\boldsymbol{v}_{*}^{\text {out }}$ at this predicted leaving point. Now we take the mean velocity

$$
\boldsymbol{v}=\frac{1}{2}\left(\boldsymbol{v}^{\text {in }}+\boldsymbol{v}_{*}^{\text {out }}\right)
$$


and considering it as a uniform velocity in the cell, calculate the master element velocity components and the corrected leaving point $\boldsymbol{P}^{\text {out }}$ as well as the propagation time.

The leaving point of the given cell is an entering point of the adjacent cell in which this algorithm is repeated. As the result, the trajectory of particle is determined by entering/leaving points at the boundary between two adjacent cells and time instants when these points are reached. After that the trajectory is linearly interpolated onto a uniform time grid.

The trajectory can be terminated

1. if the particle leaves the domain through the outlet (no adjacent cell at the leaving cell face);

2. if the particle is settled at a wall or other surface: floor, ceiling, pipeline, etc. This can occur if the particle reaches a near-boundary cell which has three boundary vertices and the velocity at the nonboundary vertex has a component toward the boundary;

3 . if the particle is trapped in the air between two adjacent cells having mean velocities directed to each other. This can happen near stagnation points in the velocity field: intersection of three flow separation surfaces. In a real flow, the probability to get into such point is infinitesimal. In a discretised domain used for computation, this probability is small but finite.

4. Some particles can remain in the air for a long time trapped by a large vortex caused by intensive ventilation.

The algorithm has been implemented in $\mathrm{C}++$. Computation of several hundred trajectories with the maximal preset time of one hour is performed in less than one second.

\section{Problem specification}

We considered an indoor space within Swansea University Bay Campus, part of a building constructed in 2018 for studying the performances of an active office [34. The air conditioning system in active office consists of two supply diffusers and one door vent as shown in Figure 1. In this work, and for the computational modelling, a human body is placed under vent2 (case 1) and at the middle between two vents (case 2) of a $46.44 \mathrm{~m}^{3}$ room $4.0 \mathrm{~m}$ by $4.3 \mathrm{~m}$, and $2.7 \mathrm{~m}$ high. Additionally, six different human standing positions $(0,60,120,180,240$, and 300 degrees $)$ are considered. Figure 1 illustrates the configuration of the simulated room. Detailed characteristics for each mesh is recorded in Table 1 for case 1 (human body under vent2). Almost the same mesh characteristics are used for case 2 (not shown). Extensive mesh sensitivity for the characteristic-based split (CBS) method has been performed in previous articles (see for example, [41, 42]). The Reynolds number for this study is defined based on the vents diameter of the room. 

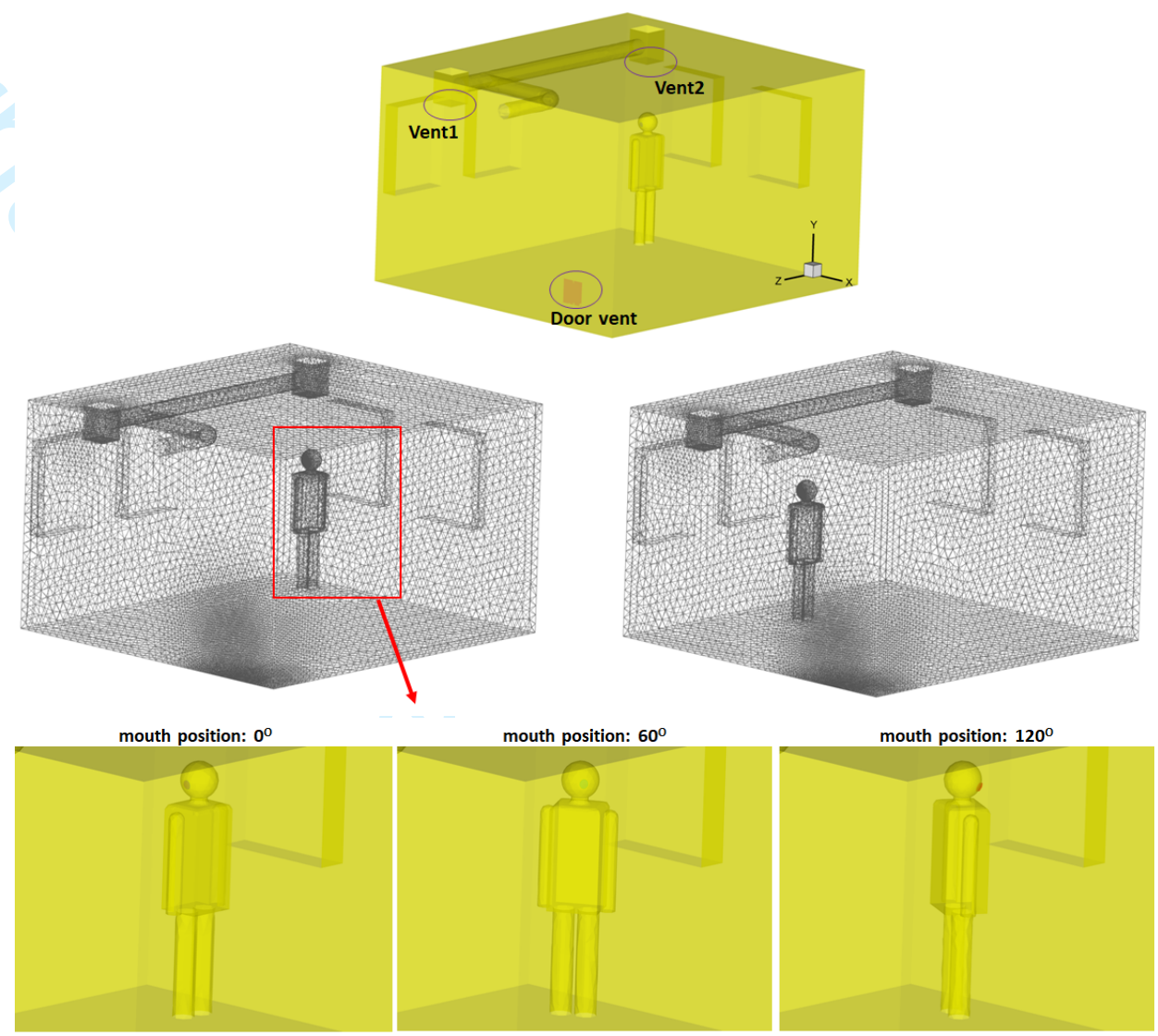

mouth position: $180^{\circ}$

mouth position: $240^{\circ}$

mouth position: $300^{\circ}$
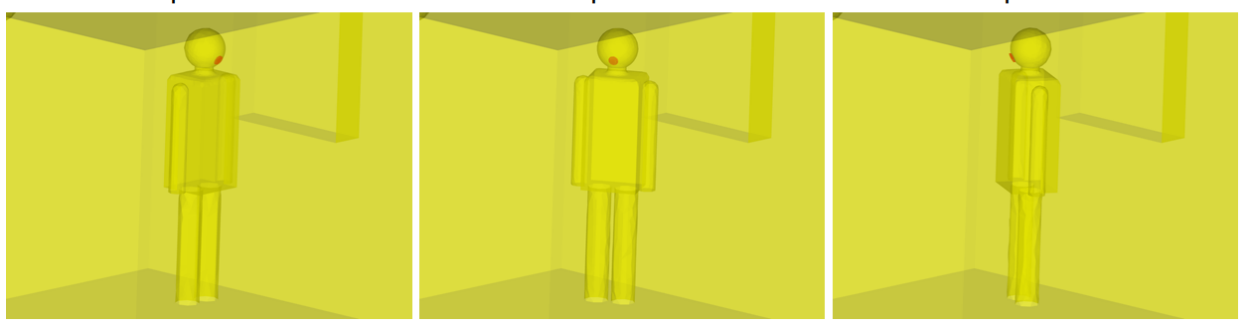

Figure 1: Schematic of model room and corresponding meshes with human standing positions

\begin{tabular}{|c|c|c|l|}
\hline $\begin{array}{c}\text { Meshes } \\
\text { (mouth positions) }\end{array}$ & Elements & Nodes & $\begin{array}{l}\text { Degree of freedom } \\
\text { (velocity, pressure, } \\
\text { turbulent eddy viscosity) }\end{array}$ \\
\hline 0-degree & 1031440 & 176625 & 883125 \\
\hline 60-degree & 1021471 & 175035 & 875175 \\
\hline 120-degree & 1021846 & 175085 & 875425 \\
\hline 180-degree & 1029153 & 176252 & 881260 \\
\hline 240-degree & 1064779 & 182161 & 910805 \\
\hline 300-degree & 1022857 & 175289 & 876445 \\
\hline
\end{tabular}

Table 1: Mesh characteristic parameters, human under vent2 


\section{Reynolds number (Re)}

\begin{tabular}{|c|c|c|c|c|c|c|c|c|c|}
\hline Vent1 & 10000 & 20000 & 30000 & 20000 & 40000 & 60000 & 0 & 0 & 0 \\
\hline Vent2 & 10000 & 20000 & 30000 & 0 & 0 & 0 & 20000 & 40000 & 60000 \\
\hline
\end{tabular}

Table 2: Reynolds numbers (Re) for different air vent diffusers

With the reference velocity of $1 \mathrm{~m} / \mathrm{s}$, a non-dimensional diffuser inlet vertical air velocity of unity is imposed. Air flow from mouth is assumed to be exhaled at a velocity inlet boundary condition $2.0 \mathrm{~m} / \mathrm{s}$ for a mouth inlet area of $0.0028 \mathrm{~m}^{2}$. No slip conditions are applied on walls and windows of the room. For the Spalart-Allmaras (SA) model the scalar variable $\hat{\nu}$ is prescribed equal to 1.0 at the inlet and zero on the solid walls. First, the steady-state solution is obtained for the airflow without particles with the convergence criterion to steady state of $10^{-6}$ tolerance value. We assumed that during a single normal breathing around $\mathrm{N}=750$ particles at the person's mouth location are released into the room.

For the ML model, a dataset containing 108 samples/numerical solution for different configurations are generated. Here, the effect of opening both vents with three different Reynolds numbers of $\mathrm{Re}=10000,20000$, and 30000 are considered. Additionally, we also considered the case in which one of the vents is closed and the flow in the other one is doubled (ie, $R e=20000$, 40000, 60000) (see Table 2). For the machine learning (see Section 4), the dataset is split randomly into train/test sets following a 80:20 ratio $(80 \%$ of the data for training and $20 \%$ of the data for testing). This database is then used to train the machine learning algorithms. The trained algorithm is then used to predict in time for 1800 seconds (30 minutes), the total number of particles in the air, the total number of particles left the room air through the vent door, the total number of particles attached to the wall, and the particles stuck in the air at locations with zero-velocity. In addition, Gated Recurrent Units (GRUs) are used to speed up the training time and to accelerate the ML workloads.

\section{Neural network architecture}

In this work, a hybrid neural networks comprising of Gated Recurrent Units (GRU) and Multi-Layer Perceptions (MLP) are used to predict the number of particles movement released from normal breathing. The GRU offers a very comparable accuracy to the more widely used Long ShortTerm Memory (LSTM), while incurring a shorter training time [43]. The GRU neural networks [26, 27, 44] is an extended and improved version of the Recurrent Neural Network (RNN) [45, 46] which are designed to work 
with the sequential data architecture and are capable of handling long-term dependencies. A RNN unit takes input from the previous step $\left(c^{<t-1>}\right)$ and current input $\left(x^{<t>}\right)$. The cell state $\left(c^{<t>}\right)$ at the current time is then given by.

$$
c^{<t>}=g\left(W_{c}\left[c^{<t-1>}, x^{<t>}\right]+b_{c}\right)
$$

where $W_{c}$ are weights, $b_{c}$ are biases, or trainable parameters, and $g$ is the activation function. RNN's face short-term memory problem and cannot process very long sequences. It is caused due to vanishing gradient problem. As RNN processes more time steps it suffers from vanishing gradient making them unable to learn long-term dependencies efficiently [47, 48].

Gated Recurrent Units (GRU) is able to process even the longest sequence data without vanishing of the gradient. GRU's are created as the solution to short-term memory. They have internal mechanisms called gates that can regulate the flow of information. As shown in Figure 2, GRU has a complex recurrent structure in a single unit, which is chronologically connected in time. GRU has two gates, reset gate $\left(\Gamma_{r}\right)$ and update gate $\left(\Gamma_{u}\right)$. The reset gates are used to decide how much past knowledge are irrelevant later in the future to drop and update gates and decide what knowledge to be added to the cell state. Each gate has its own weights and biases. The relationship between the input and the output of GRU may be defined by a set of the following equations:

$$
\begin{array}{r}
\hat{c}^{<t>}=\tanh \left(W_{c}\left[\Gamma_{r} * c^{<t-1>}, x^{<t>}\right]+b_{c}\right) \\
\Gamma_{r}=\sigma\left(W_{r}\left[c^{<t-1>}, x^{<t>}\right]+b_{r}\right) \\
\Gamma_{u}=\sigma\left(W_{u}\left[c^{<t-1>}, x^{<t>}\right]+b_{u}\right) \\
c^{<t>}=\Gamma_{u} * \hat{c}^{<t>}+\left(1-\Gamma_{u}\right) * c^{<t-1>}
\end{array}
$$

where $W_{c}, W_{r}$, and $W_{u}$ are the parameter matrices (weights) and $b_{c}, b_{r}$, and $b_{u}$ are bias vectors. $\sigma(x)=\frac{1}{1+\exp (-x)}$ is the element-wise logistic sigmoid function. The corresponding diagram for equations (16) is displayed in Figure 2 .

The main part of the Artificial Neural Networks methodology is the learning or training process in which the errors determined at the output layer are successively reduced by adjusting the weights and biases throughout the network. The back-propagation algorithm changes the weights towards a lower error at the end. The network weights and biases of Neural Networks (NNs) are tuned based on data using the adaptive moment estimation (Adam) [49] algorithm. Adam method is one of the most popular gradient-based optimization algorithms for optimizing neural networks and is computationally efficient, has little memory requirement, and is well suited for problems that are large in terms of data/parameters [49. In this 


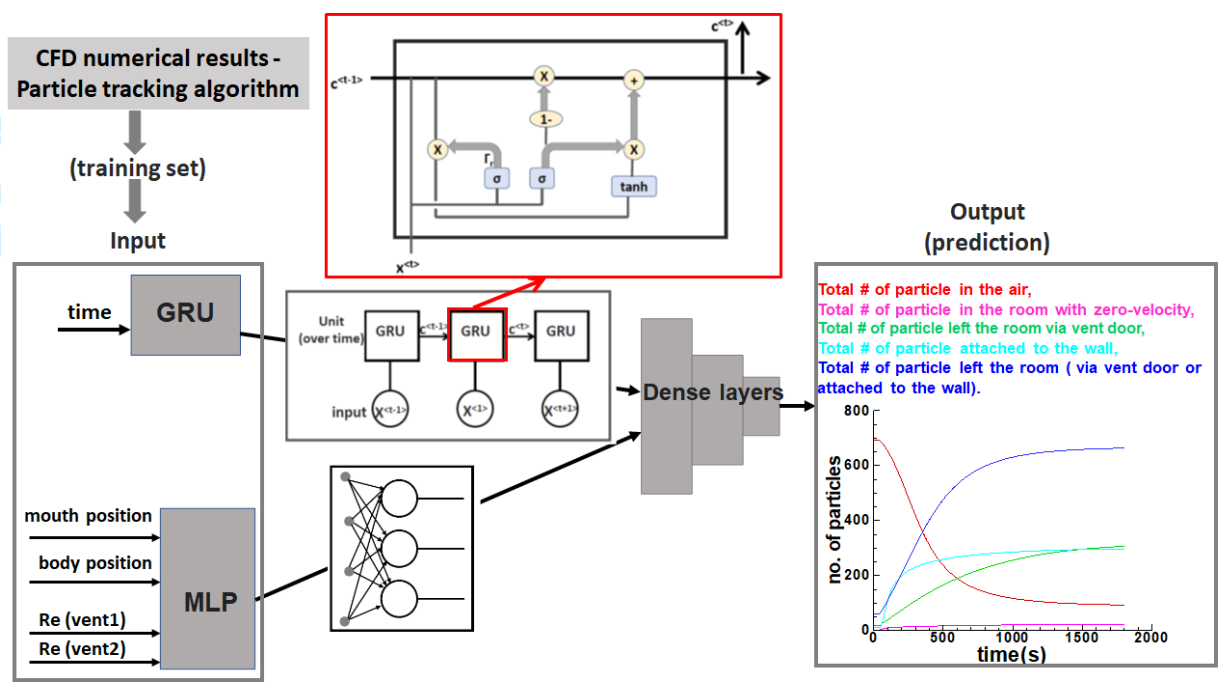

Figure 2: The diagram of the hybrid neural networks comprising of Gated Recurrent Units (GRU) and Multi-Layer Perceptions (MLP), input and output data

work, default setting of hyper-parameters of Adam optimization algorithm are used. For more details on Adam optimizer see [49, 50].

The input to Neural Networks are inlet vent conditions, location of a person in the room with different mouth positions and particle dispersion and deposition. The output of the NN is the total number of particles in the air, the total number of particles left the room through the outlet vent door, the total number of particles attached to the wall, and the particles stuck in the air at locations with zero-velocity. The detailed network configuration and the parameters, the input to Neural Networks, and the output of the NN used in this work are shown Figure 2 and in Table 3. In order to verify the prediction accuracy performance of the proposed model for the continuous-time associated to our airborne movement problem, this paper uses the mean square error (MSE) as the model criterion, ie,

$$
E_{M S E}=\frac{1}{n} \sum_{i=1}^{n}\left(x_{i}-\hat{x}_{i}\right)^{2}
$$

where $\hat{x}_{i}$ is the prediction value and $n$ is the number of sample points in the test data set.

\section{Results and Discussion}

In the present work we analysed three main factors affecting the particle transport: air flow ventilation rates, human standing positions, and human location in the room. In the proposed settings, variations in the ventilation 
Table 3: ANN parameters - airborne movement problem

\section{Gated Recurrent Unit \\ 2-4 \\ 2 or 4}

2: with 11 and 8 neurons

Number of dense layers

Number of fully connected layers

Dense activation layers

Output activation layer

loss function

Number of epochs

Validation-split

Optimiser

1

1 (output layer, no. of particles in time)

Tanh (hyperbolic tangent)

Sigmoid function

Mean squared error

1000

0.2

Adam (default hyper-parameters)

air flow (ie, Re=10000-60000), human standing positions (0, 60, 120, 180, 240 , and 300 degrees), and human location in the room significantly modified the level of air flow and airborne infections response.

\subsection{Effects of ventilation rates}

The role of ventilation rates in airborne infections are shown in Figure 3 for three different vents scenarios where either both vents are open or one of the vents is closed and the Reynolds number on the other vent is doubled. The general trends demonstrate how the infection can be persistently carried by the airflow in the room from one point to another depending on the pattern of the airflow, location of ventilators, and location of the human. In Figure 3, steady state visual representation of airflow trajectories (stream wise, top) and contour plot (middle) colored to velocity magnitude is presented for $R e=20000$ (both vents open), $R e=40000$ (vent1: open, vent2: closed), and $\operatorname{Re}=40000$ (vent1: closed, vent2: open) with the human standing position of 0 degree (see Figure 1). Additionally, in Figure 3 (bottom), the distribution of particles released from normal breathing is displayed in time for 1800 seconds (30 minutes). The red line indicates the total number of particles in the air. Blue line shows the total number of particles left the room air through the vent door [green] or attached to the wall [cian]. The particles stuck in the air at locations with zero-velocity are shown in magenta color. It is clearly apparent that when vent 1 is closed and vent 2 is open (Figure 3. f), the total number of particles left the room air $(\mathrm{O}(400)$, $55 \%$ of the particles, blue line) is less than the case when vent 1 is open and vent2 is closed $(\mathrm{O}(660)$, almost $90 \%$ of the particles, (Figure 3b, e)). This is due to closing the air vent where some particles remain under vent1 and not moving. Furthermore, when both vents are open (Figure 3 a, d), the total number of the particles without interacting with any surfaces $(55 \%$, 

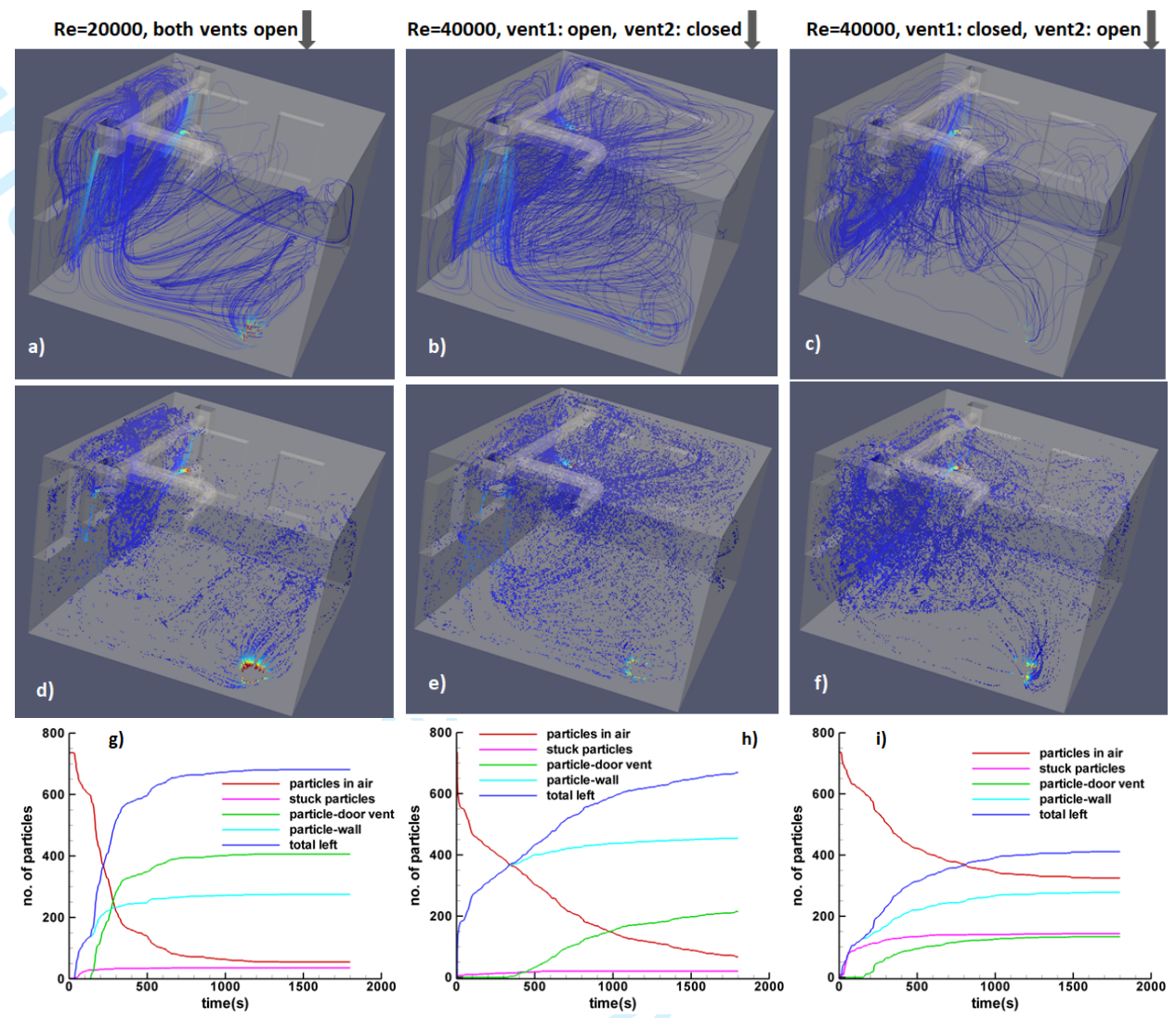

Figure 3: Steady state visual representation of airflow trajectories in active office colored to velocity magnitude (a-f), Distribution of particles released from normal breathing (g-l) in time, human under vent 2 , mouth position: 0 degee, $R e=20000,40000$

Figure 3g) left the room after 30 minutes (green line) are greater than the cases when one of the vents is open (around $30 \%$ and $16 \%$, Figures $3 \mathrm{~h}$, i, green lines). In general, when both vents are open, the particles in air (red line) move rapidly comparing with the cases with only one vent open.

Next, we compare the performance of the hybrid neural network prediction model described in Section 4 with the the numerical results obtained using the CFD model. Figure 5 shows the model loss for the training dataset for Gated Recurrent Unit(GRU) with different level of layers and neurons. Figure 4 shows the CFD-data against the machine learning predictions with three GRU model variants, GRU(4-2), 2 layers with 4 and 2 units, GRU(42-2), 3 GRU layers with 4, 2 and 2 units, and GRU(4-2-2-2), 4 layers with 4, 2, 2 and 2 units, respectively. In Figure 4 , the total number of particles in the air, the particles stuck in the air at locations with zero-velocity, and the total number of particles left the room air through the vent door are captured in 1800 seconds (30) minutes. In this case the values of Reynolds number are taken to be $\operatorname{Re}=40000$ (both vents are open, Figure 4 a) and 

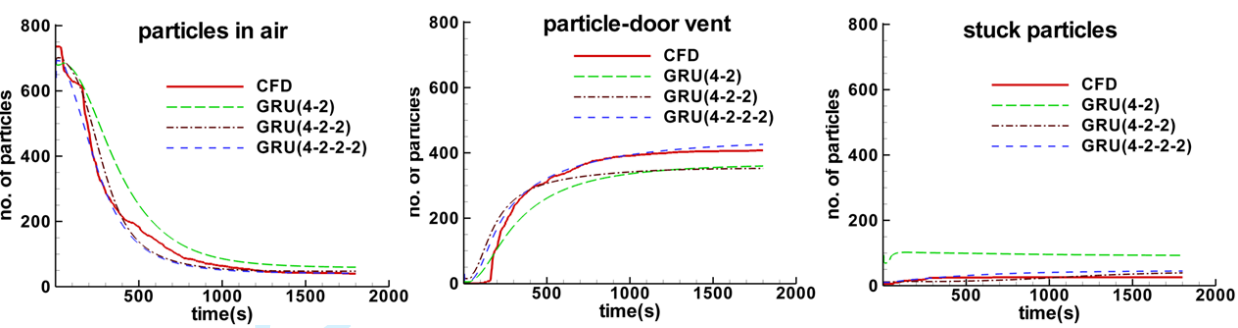

(a)

Re=50000, Vent1 closed, Vent 2 open
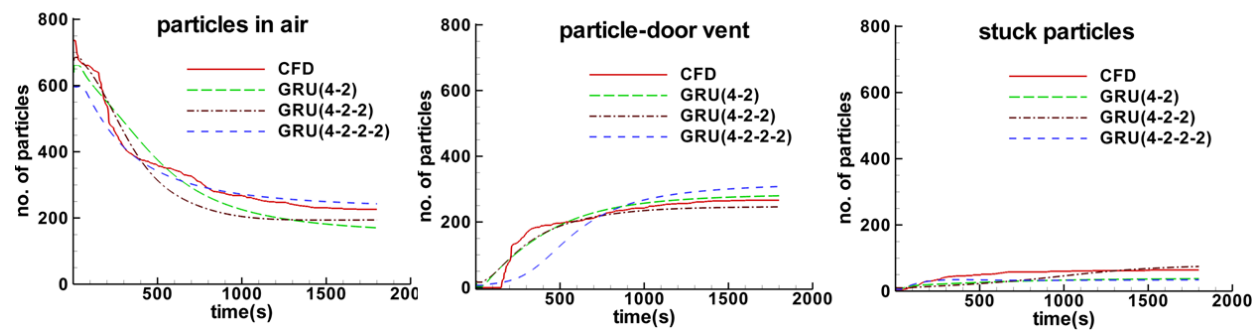

(b)

Figure 4: Distribution of particles released from normal breathing in time, human under vent 2 , mouth position: 0 degree, a) $R e=40000$,both vents open b) $R e=50000$, vent 1 closed, vent2 open, CFD vs Gated Recurrent Unit(GRU) with different level of layers and units

Re=50000 (vent1 closed, vent2 open, Figure 4b). Note that, both Reynolds number values are outside the training data range. As shown in Figures $4 \mathrm{a}$ and $4 \mathrm{~b}$, the CFD results (red lines) are captured in a tight window provided by the range of GRUs neural networks (GRU(4-2-2), GRU(4-2), and GRU(4-2)). Here, GRU(4-2-2-2) with more number of trainable parameters presents a closer prediction to CFD findings when compared to its counterpart GRU(4-2).

In addition, model loss for the training dataset for GRU(4-2), GRU(42-2), and GRU(4-2-2-2) are shown in Figure 5. The loss values predicted by the neural network for trained data are around $1.5 \%$ for almost all GRUs, after an initial stabilisation period. Furthermore, very little differences in training loss are observed between the three GRU neural networks. Additionally, the training loss is consistent with increasing number of epochs. This leads to model stability and non overfitting, indicating desirable model performance. 


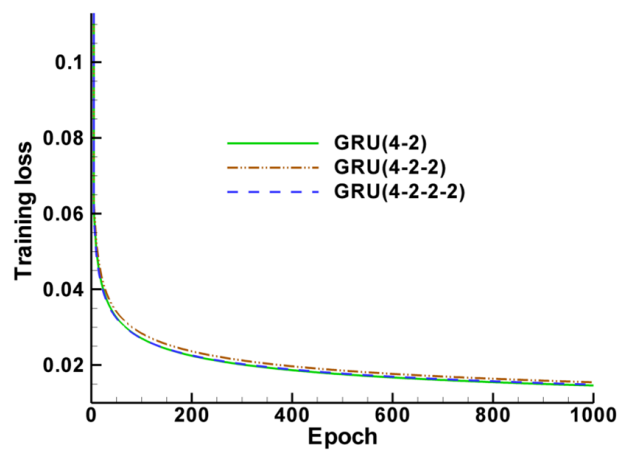

Figure 5: Model loss for the training dataset for Gated Recurrent Unit(GRU) with different level of layers and neurons

\subsection{Effects of human standing position}

The overall impact of human position on air flow trajectories and particle distribution for selected three different human standing positions of 0,240 , and 300 degrees is presented in Figure 6. In this case, Reynolds number is taken to be $\mathrm{Re}=30000$ where both vents are open. In Figure 6c and its counterpart GRUNN-prediction plot in Figure 6f, one may note the rapid decline in number of particles in air (red line) and at the same time rapid increase in the number of particles left the room (blue line). This is due to orientation and mouth position of 300 degree, where the human standing position is in a direction toward the vent 1 and this causes the particles to leave the room much faster when comparing to other cases with human orientation in the room. In this case (mouth position: 300), almost $97 \%$ of the total particles (Figure 6f, blue line) left the room after around 8 minutes (500 seconds). Yet, The total number of particles left the room at the same time (8 minutes) with human standing positions of 0 , and 240 degrees are approximately $66 \%$, and $90 \%$, respectively ((Figures 6 d, e). Moreover, the number of particles attached to the wall [cian] is almost $16 \%$ of the total released particles for the case of 300 degree mouth position after 30 minutes (1800 second), (Figure 6f). The number of particles attached to the wall is increased to around $38 \%$, and $40 \%$ of the total particles, for 240, and 0 degree mouth position cases, respectively (Figures 6e, d). Furthermore, the maximum number of particles remain in suspension in the system after 30 minutes (1800 seconds) are around 17 particles (4\% of the total particles) for the case of 0 degree mouth position (Figure 6 $\mathrm{d}$ ).

\subsection{Effects of human location in room}

In this section, prediction under two different human locations (human under vent 2 and human in the middle of the room between two vents) are considered, with the purpose of investigating the effects of movement and maintaining social distancing from the infectious person in the room and 

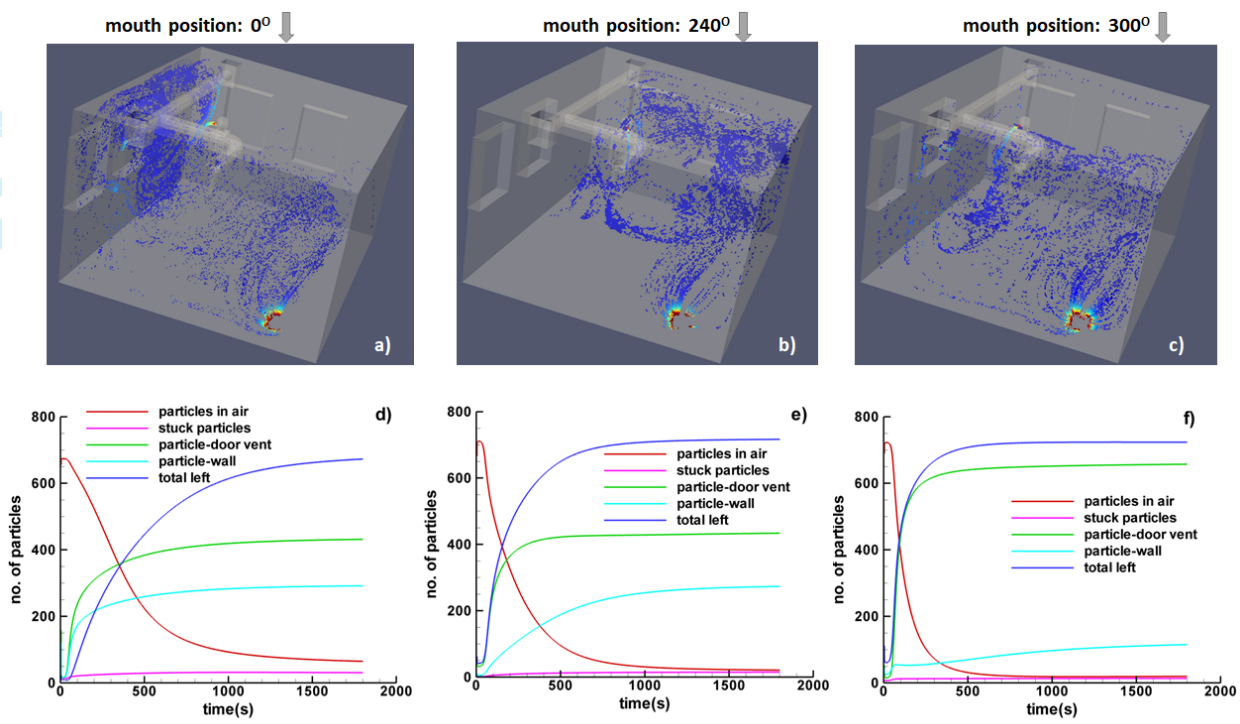

Figure 6: Steady state visual representation of airflow trajectories in active office colored to velocity magnitude (a-c), human under vent $2, \mathrm{Re}=30000$, prediction of particles movement released from normal breathing (d-f) in time, GRU(4-2-2-2)

predicting of particle movement released from normal breathing. In Figure $7 \mathrm{a}-\mathrm{d}$, steady state visual representation of airflow trajectories is presented for $R e=30000$ with the human standing position of 120 , and 180 degrees. In addition, for the same setting, the Neural network for prediction of number of particles movement in time is shown in Figure 77-h. As expected, the particles spread more through the space when the human is in the middle of the room between the vents (Figure $7 \mathrm{k}, \mathrm{d}$ ) compared to the case when the human is at the corner of the room under vent2 (Figure $7 \mathrm{a}, \mathrm{b}$ ). Considering the orientation and mouth position of 180 degree with human in the middle of the room (Figure $7 \mathrm{~h}$ ), a sharp drop in particle movement in air (red line) is evident when comparing to its counterpart in Figure 7f with human at the corner of the room under vent2. Moreover, almost $40 \%$ of the particles left the room air directly through the vent door (Figure 78,f, green lines) whilst this increases to $68 \%$ in the case with human in the middle of the room between vents (Figure $7 \mathrm{~g}, \mathrm{~h}$ ). Additionally, Figure $7 \mathrm{~h}$ with human in the middle between vents and with mouth position of 180 degree reports lowest number of particles (13\%) attached to the wall (cian line).

Finally, Figure 8 demonstrates that the Gated Recurrent Units Neural Network (GRU-NN) is capable of predicting the aerosol movement under various ventilation conditions with different human mouth position. Note that, all the inputs for the GRU-NN prediction model in Figure $8 \mathrm{a}-\mathrm{f}$ are taken to be outside the training data range. 

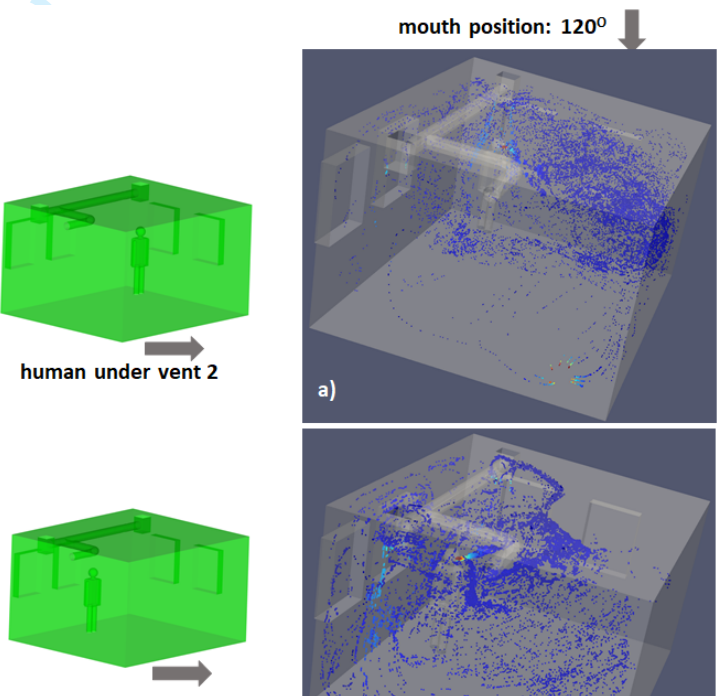

human in the middle between vents
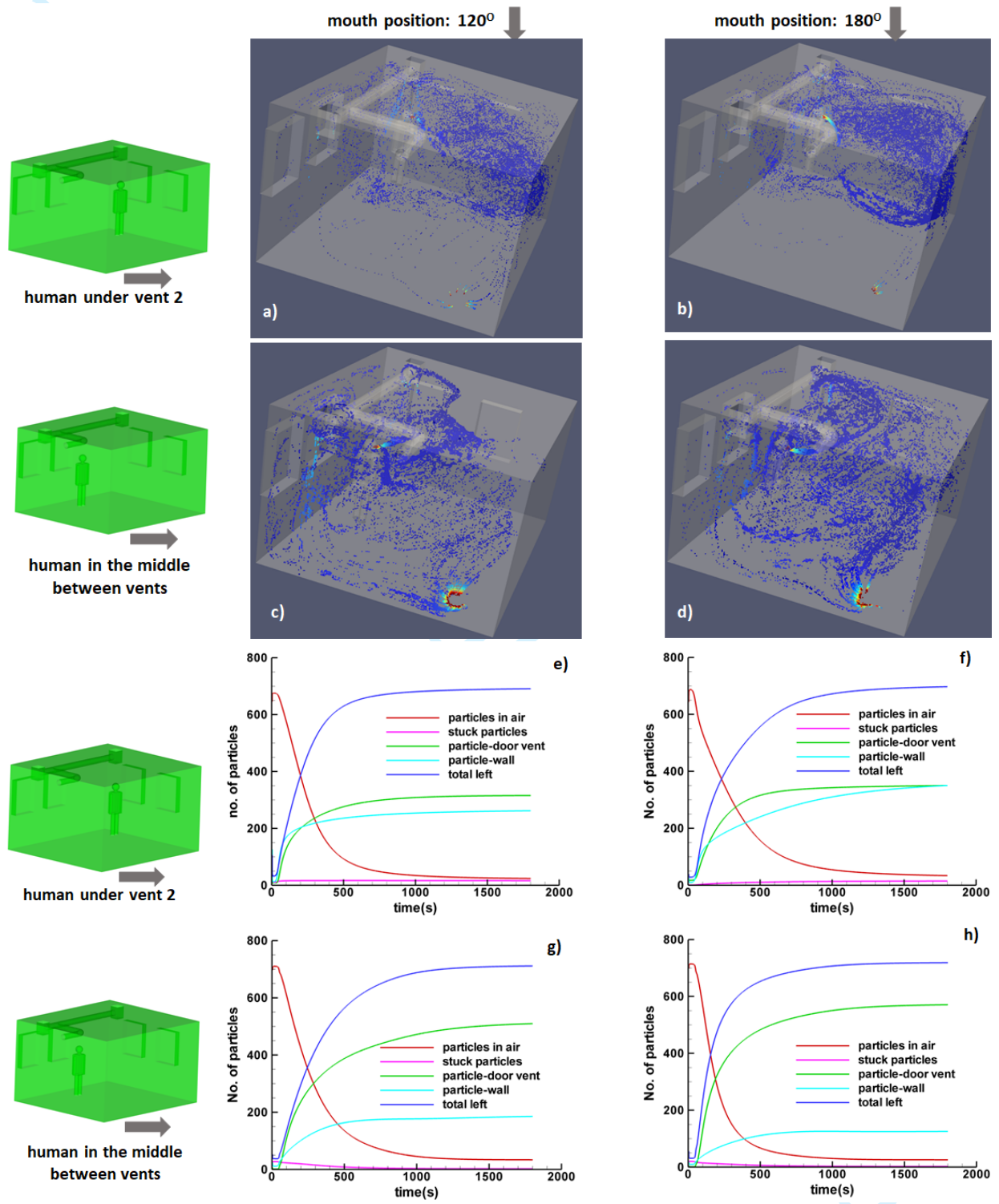

Figure 7: Steady state visual representation of airflow trajectories in active office colored to velocity magnitude, human under vent2 $(\mathrm{a}, \mathrm{b})$, human in the middle between vents $(\mathrm{c}, \mathrm{d}), \mathrm{Re}=30000$, prediction of particles movement released from normal breathing (d-f) in time, GRU(4-2-2-2), human under vent2 (e,f), human in the middle between vents (g,h) 

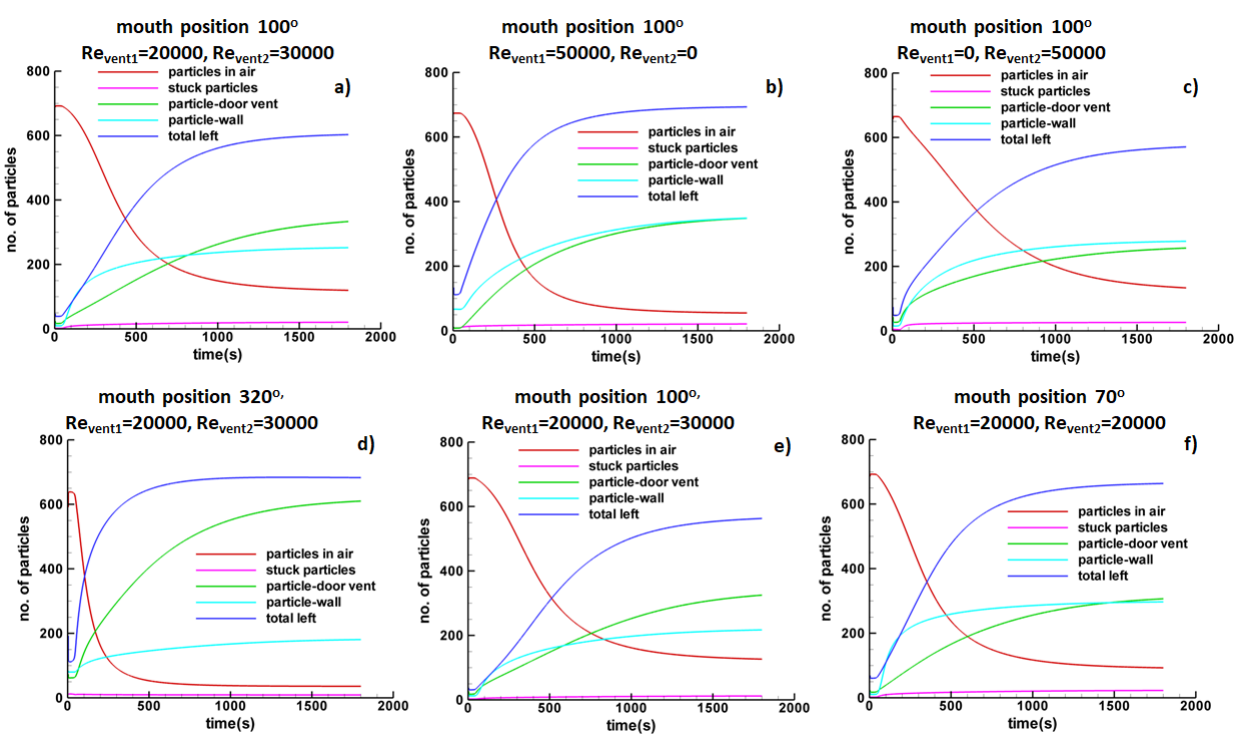

Figure 8: Prediction of particles movement released from normal breathing (a-f) in time with GRU(4-2-2-2), Various Re and human mouth positions

\section{Conclusions}

Through this work a predictive method for the accurate capture of infectious particle behaviours, originated during normal breathing of a human, is established. The methodology is based on a Gated Recurrent Units Neural Network (GRUs-NN) model, which is capable of handling long-term dependencies. High-fidelity prediction of indoor air flow is obtained by means of an in-house parallel CFD solver which employs a one equation Spalrat-Allmaras (SA) turbulence model. Several flow scenarios regarding the operation of inlet vents, location of a person in an active office with different human standing positions and particle dispersion and deposition in the enclosed environment are considered. The airflow pattern shows how the particles can be carried by the airflow within active office. The recorded airflow pattern in the active office can be very complex, depending on the location of air ventilations and ventilation rates, as well as on the position of particles. Movement of people within the room can further complicate the resulting fluid dynamics. Through the proposed cases we showed that the Gated Recurrent Units Neural Network model can provide predictions in time which are in good agreement with the CFD numerical results. This study paves the way for the development of efficient and reliable tools for predicting virus airborne movement under different ventilation conditions and different human positions within an indoor environments, potentially leading to new design. 


\section{Acknowledgements}

Authors acknowledge the financial support from Ser Cymru III - Tackling Covid 19 fund, Welsh Government Project number 095. The authors are also grateful for helpful discussion with Dr Justin Searle and active office team, SPECIFIC Innovation \& Knowledge Centre, Swansea University, UK.

\section{References}

[1] Michael A Kohanski, L James Lo, and Michael S Waring. Review of indoor aerosol generation, transport, and control in the context of COVID-19. In International forum of allergy \& rhinology, volume 10, pages 1173-1179. Wiley Online Library, 2020.

[2] Neeltje Van Doremalen, Trenton Bushmaker, Dylan H. Morris, Myndi G. Holbrook, Amandine Gamble, Brandi N. Williamson, Azaibi Tamin, Jennifer L. Harcourt, Natalie J. Thornburg, Susan I. Gerber, et al. Aerosol and surface stability of SARS-CoV-2 as compared with SARS-CoV-1. New England Journal of Medicine, 382(16):1564-1567, 2020.

[3] Lidia Morawska and D.K. Milton. It Is Time to Address Airborne Transmission of Coronavirus Disease 2019 (COVID-19). Clinical Infectious Diseases, 71(9):2311-2313, 2020.

[4] Mengjia Tang, Ningling Zhu, Kerry Kinney, and Atila Novoselac. Transport of indoor aerosols to hidden interior spaces. Aerosol Science and Technology, 54(1):94-110, 2020.

[5] Ramon Codina, Christopher Morton, Eugenio Onate, and Orlando Soto. Numerical aerodynamic analysis of large buildings using a finite element model with application to a telescope building. International Journal of Numerical Methods for Heat \& Fluid Flow, 10(6):613-633, 2000 .

[6] Souad Morsli, Mustapha Boussoufi, Amina Sabeur, Mohammed El Ganaoui, and Rachid Bennacer. Small to large scale mixed turbulent convection: buildings application. International Journal of Numerical Methods for Heat \& Fluid Flow, 28(1):188-205, 2018.

[7] Zohir Younsi, Lounes Koufi, and Hassane Naji. Numerical study of the effects of ventilated cavities outlet location on thermal comfort and air quality. International Journal of Numerical Methods for Heat E Fluid Flow, 29(11):4462-4483, 2019. 
[8] Pedro M de Oliveira, Leo CC Mesquita, Savvas Gkantonas, Andrea Giusti, and Epaminondas Mastorakos. Evolution of spray and aerosol from respiratory releases: theoretical estimates for insight on viral transmission. Proceedings of the Royal Society A, 477(2245):20200584, 2021.

[9] Khaled Talaat and Jinxiang Xi. Computational modeling of aerosol transport, dispersion, and deposition in rhythmically expanding and contracting terminal alveoli. Journal of Aerosol Science, 112:19-33, 2017.

[10] Vinh Van Tran, Duckshin Park, and Young-Chul Lee. Indoor air pollution, related human diseases, and recent trends in the control and improvement of indoor air quality. International Journal of Environmental Research and Public Health, 17(8):2927, 2020.

[11] Ioannis Manisalidis, Elisavet Stavropoulou, Agathangelos Stavropoulos, and Eugenia Bezirtzoglou. Environmental and health impacts of air pollution: A review. Frontiers in public health, 8, 2020.

[12] Luis Alfredo Anchordoqui and Eugene M Chudnovsky. A Physicist View of COVID-19 Airborne Infection through Convective Airflow in Indoor Spaces. SciMedicine Journal, 2:68-72, 2020.

[13] D Fontes, J Reyes, K Ahmed, and M Kinzel. A study of fluid dynamics and human physiology factors driving droplet dispersion from a human sneeze. Physics of Fluids, 32(11):111904, 2020.

[14] Sanjay Kumar and Heow Pueh Lee. The perspective of fluid flow behavior of respiratory droplets and aerosols through the facemasks in context of SARS-CoV-2. Physics of Fluids, 32(11):111301, 2020.

[15] Ville Vuorinen, Mia Aarnio, Mikko Alava, Ville Alopaeus, Nina Atanasova, Mikko Auvinen, Nallannan Balasubramanian, Hadi Bordbar, Panu Erästö, Rafael Grande, et al. Modelling aerosol transport and virus exposure with numerical simulations in relation to SARSCoV-2 transmission by inhalation indoors. Safety Science, 130:104866, 2020 .

[16] Rainald Löhner and Harbir Antil. High fidelity modeling of aerosol pathogen propagation in built environments with moving pedestrians. International Journal for Numerical Methods in Biomedical Engineering, 37(3):e3428, 2021.

[17] Rainald Löhner, Harbir Antil, Sergio Idelsohn, and Eugenio Oñate. Detailed simulation of viral propagation in the built environment. Computational Mechanics, 66(5):1093-1107, 2020. 
[18] Mohamed Abuhegazy, Khaled Talaat, Osman Anderoglu, and Svetlana V Poroseva. Numerical investigation of aerosol transport in a classroom with relevance to COVID-19. Physics of Fluids, 32(10):103311, 2020 .

[19] Zechariah Lau, Katerina Kaouri, and Ian M Griffiths. Modelling Airborne Transmission of COVID-19 in Indoor Spaces Using an AdvectionDiffusion-Reaction Equation. arXiv preprint arXiv:2012.12267, 2020.

[20] Z Lau, IM Griffiths, A English, and K Kaouri. Predicting the Spatially Varying Infection Risk in Indoor Spaces Using an Efficient Airborne Transmission Model. 2021.

[21] Yiping Li, Gabriel M Leung, JW Tang, Xiaozhan Yang, CY Chao, John Zhang Lin, JW Lu, Per Væggemose Nielsen, Jianlei Niu, H Qian, et al. Role of ventilation in airborne transmission of infectious agents in the built environment-a multidisciplinary systematic review. Indoor air, 17(1):2-18, 2007.

[22] Shengwei Zhu, Sara Jenkins, Kofi Addo, Mohammad Heidarinejad, Sebastian A Romo, Avery Layne, Joshua Ehizibolo, Daniel Dalgo, Nicholas W Mattise, Filbert Hong, et al. Ventilation and laboratory confirmed acute respiratory infection (ARI) rates in college residence halls in College Park, Maryland. Environment international, 137:105537, 2020.

[23] Hua Qian and Xiaohong Zheng. Ventilation control for airborne transmission of human exhaled bio-aerosols in buildings. Journal of thoracic disease, 10(Suppl 19):S2295, 2018.

[24] Tom Lipinski, Darem Ahmad, Nicolas Serey, and Hussam Jouhara. Review of ventilation strategies to reduce the risk of disease transmission in high occupancy buildings. International Journal of Thermofluids, page $100045,2020$.

[25] Yi Luo, Liehui Zhang, Yin Feng, and Yulong Zhao. Three-Dimensional Streamline Tracing Method over Tetrahedral Domains. Energies, $13(22): 6027,2020$.

[26] Kyunghyun Cho, Bart Van Merriënboer, Dzmitry Bahdanau, and Yoshua Bengio. On the properties of neural machine translation: Encoder-decoder approaches. arXiv preprint arXiv:1409.1259, 2014.

[27] Junyoung Chung, Caglar Gulcehre, K Cho, and Y Bengio. Empirical evaluation of gated recurrent neural networks on sequence modeling. arXiv 2014. arXiv preprint arXiv:1412.3555, 2014. 
[28] Sumit Das, Aritra Dey, Akash Pal, and Nabamita Roy. Applications of artificial intelligence in machine learning: review and prospect. International Journal of Computer Applications, 115(9), 2015.

[29] Devanshi Dhall, Ravinder Kaur, and Mamta Juneja. Machine learning: a review of the algorithms and its applications. Proceedings of ICRIC 2019, pages 47-63, 2020.

[30] Yoshua Bengio. Learning deep architectures for AI. Foundations and trends(R) in Machine Learning, 2(1):1-127, 2009.

[31] Capuano, German and Rimoli, Julian J. Smart finite elements: A novel machine learning application. Computer Methods in Applied Mechanics and Engineering, 345:363-381, 2019.

[32] Tamaddon-Jahromi, Hamid Reza and Chakshu, Neeraj Kavan and Sazonov, Igor and Evans, Llion M and Thomas, Hywel and Nithiarasu, Perumal. Data-driven inverse modelling through neural network (deep learning) and computational heat transfer. Computer Methods in Applied Mechanics and Engineering, 369:113217, 2020.

[33] Rainald Löhner, Harbir Antil, Hamid Tamaddon-Jahromi, Neeraj Kavan Chakshu, and Perumal Nithiarasu. Deep learning or interpolation for inverse modelling of heat and fluid flow problems? International Journal of Numerical Methods for Heat \& Fluid Flow, 2021.

[34] J. Clarke. Active Classroom Case Study. Technical report, Architectural Design Manager, SPECIFIC, Version 2.0, 2020.

[35] Philippe Spalart and Steven Allmaras. A one-equation turbulence model for aerodynamic flows. In 30th aerospace sciences meeting and exhibit, page 439, 1992.

[36] Steven R Allmaras and Forrester T Johnson. Modifications and clarifications for the implementation of the spalart-allmaras turbulence model. In Seventh international conference on computational fluid $d y$ namics (ICCFD'), pages 1-11, 2012.

[37] P. Nithiarasu and C. B. Liu. An artificial compressibility based characteristic based split (cbs) scheme for steady and unsteady turbulent incompressible flows. Computer Methods in Applied Mechanics and Engineering, 195(23-24):2961-2982, 2006.

[38] HamidReza Tamaddon Jahromi, Samuel Rolland, Jason Jones, Alberto Coccarelli, Igor Sazonov, Chris Kershaw, Chedly Tizaoui, Peter Holliman, David Worsley, Hywel Thomas, et al. Modelling ozone disinfection process for creating COVID-19 secure spaces. International Journal of Numerical Methods for Heat \& Fluid Flow, 2021. 
[39] Zienkiewicz, OC and Taylor, RL and Nithiarasu, P. Introduction to the equations of fluid dynamics and the finite element approximation. The Finite Element Method for Fluid Dynamics. 7th ed. Oxford: Elsevier Butterworth-Heinemann, pages 1-29, 2014.

[40] P Nithiarasu, JS Mathur, NP Weatherill, and K Morgan. Threedimensional incompressible flow calculations using the characteristic based split (CBS) scheme. International Journal for Numerical Methods in Fluids, 44(11):1207-1229, 2004.

[41] Codina, Ramon and Coppola-Owen, H and Nithiarasu, P and Liu, CB. Numerical comparison of CBS and SGS as stabilization techniques for the incompressible Navier-Stokes equations. International Journal for Numerical Methods in Engineering, 66(10):1672-1689, 2006.

[42] Nithiarasu, P and Liu, C-B and Massarotti, N. Laminar and turbulent flow calculations through a model human upper airway using unstructured meshes. Communications in Numerical Methods in Engineering, 23(12):1057-1069, 2007.

[43] Rajib Rana. Gated recurrent unit (GRU) for emotion classification from noisy speech. arXiv preprint arXiv:1612.07r78, 2016.

[44] Gail Weiss, Yoav Goldberg, and Eran Yahav. On the practical computational power of finite precision rnns for language recognition. arXiv preprint arXiv:1805.04908, 2018.

[45] Rumelhart, David E and Hinton, Geoffrey E and Williams, Ronald J. Learning representations by back-propagating errors. nature, 323(6088):533-536, 1986.

[46] Erdenebayar Urtnasan, Jong-Uk Park, and Kyoung-Joung Lee. Automatic detection of sleep-disordered breathing events using recurrent neural networks from an electrocardiogram signal. Neural computing and applications, 32(9):4733-4742, 2020.

[47] Lechner, M and Hasani, R. Learning Long-Term Dependencies in Irregularly-Sampled Time Series. arXiv 2020. arXiv preprint arXiv:2006.04418.

[48] Ribeiro, Antônio H and Tiels, Koen and Aguirre, Luis A and Schön, Thomas. Beyond exploding and vanishing gradients: analysing RNN training using attractors and smoothness. In International Conference on Artificial Intelligence and Statistics, pages 2370-2380. PMLR, 2020.

[49] Adam D.P. Kingma, J. Ba. A method for stochastic optimization. Anon. International Conferenceon Learning Representations. SanDego: ICLR, 2015. 
[50] Sashank J Reddi, Satyen Kale, and Sanjiv Kumar. On the convergence of adam and beyond. arXiv preprint arXiv:1904.09237, 2019. 


\section{Reply to Reviewer 1:}

We acknowledge the comments offered by Reviewer 1, and summarise the detailed corrections within the response below:

- As requested, more theoretical details of the Deep Neural Networks have now been added to the revised version of the paper in pages 9-11. This includes the following figure (Figure 2 page 10) in which the detailed explanation of the input and output data are included.

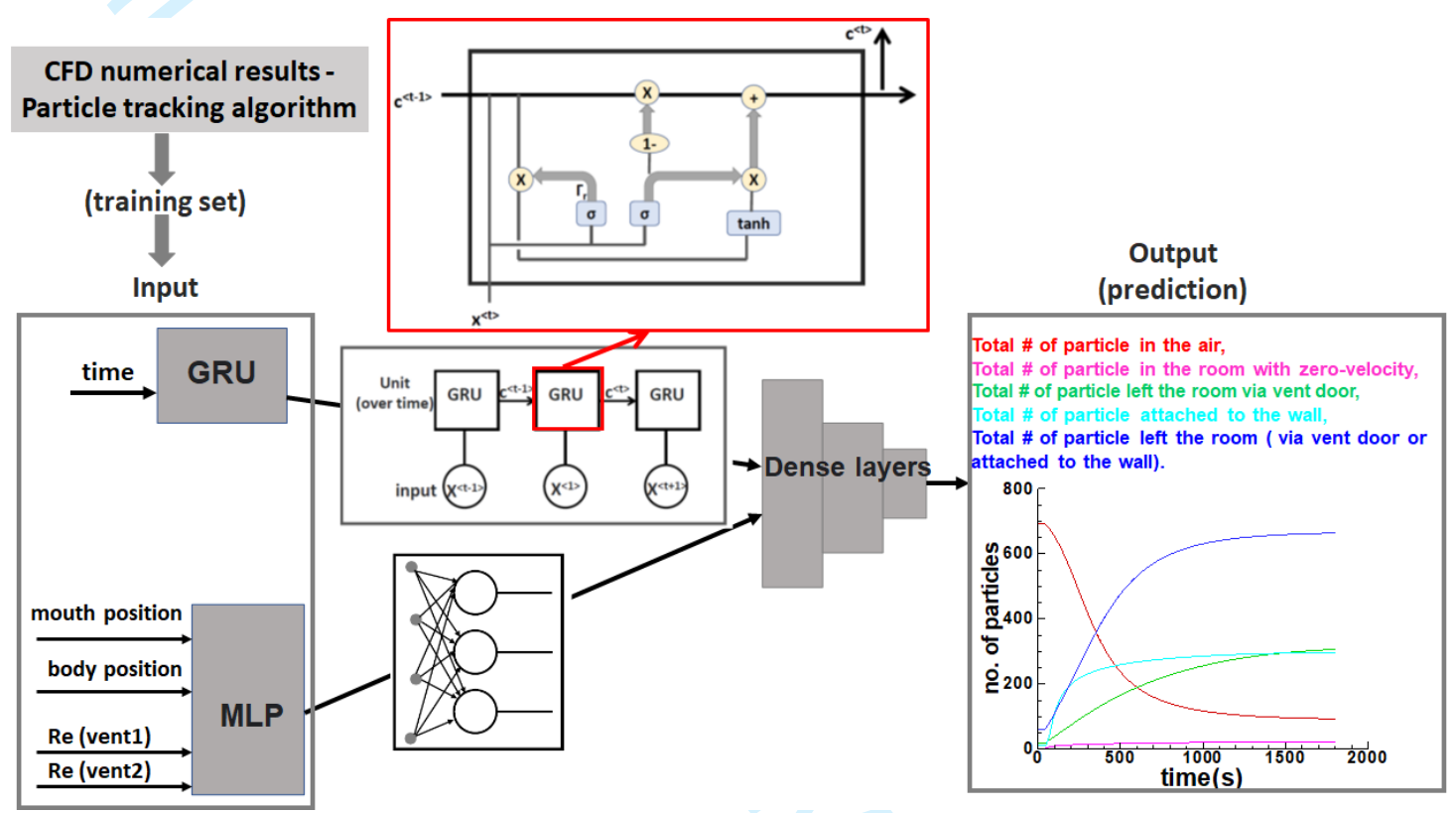

- More details of the loss function are added to the manuscript. In addition, a new figure (Figure 5) for model loss for the training dataset is included. Please see pages 10,13 , and 14.

- More details of the Adam optimization algorithm are added to the manuscript. Please see page 9.

- More details of neural network hyper-parameters are included in Table 3 (page 11).

- We believe that with the new DNN details added in the paper, the results are reproducible.

We hope that these detailed corrections and responses clarify the various points raised by this reviewer, whom we thank for his constructive comments. 